\title{
Spectra of electronic excitations in graphene near Coulomb impurities
}

\author{
A. I. Breev ${ }^{1 *}$, R. Ferreira ${ }^{2 \dagger}$ D. M. Gitman ${ }^{1,2,3 \ddagger}$ and B. L. Voronov ${ }^{3 \S}$ \\ ${ }^{1}$ Department of Physics, Tomsk State University, Tomsk 634050, Russia; \\ ${ }^{2}$ Institute of Physics, University of São Paulo, CEP 05508-090, São Paulo, SP, Brazil; \\ ${ }^{3}$ P.N. Lebedev Physical Institute, 53 Leninsky prospekt, 119991 Moscow, Russia;
}

August 6, 2021

\begin{abstract}
We study the problem of the electron excitation spectrum in the presence of point-like and regularized Coulomb impurities in gapped graphene. To this end, we use the Dirac model and in the point-like case theory of self-adjoint extensions of symmetric operators. In the point-like case, we construct a family of self-adjoint Hamiltonians describing the excitations for any charge of an impurity. Spectra and (generalized) eigenfunctions for all such Hamiltonians are found. Then, we consider the spectral problem in the case of a regularized Coulomb potential of impurities for a special regularization. We study exact equations for charges of impurities that may generate bound states with energy that coincides with the upper boundary of the negative branch of the continuous spectrum (supercritical charges) and calculate these charges.
\end{abstract}

\section{Introduction}

It is well known that low-energy electronic excitations in the graphene monolayer in the presence of an external electromagnetic field can be described by the Dirac model with the corresponding background [1, namely, by a $2+1$ quantized Dirac field in such a background. In fact, in the framework of the latter model, it is more correct to speak about some quasiparticles, which are chiral Dirac fermions in $2+1$ dimensions. Taking into account that dispersion surfaces are the so-called Dirac cones, the gap between the upper and lower branches in the corresponding Dirac particle spectra is very small and charge carriers are massless, we can expect that Schwinger

\footnotetext{
*breev@mail.tsu.ru

${ }^{\dagger}$ rafaelufpi@gmail.com

¥ gitman@if.usp.br

§oronov@lpi.ru
} 
mechanism of vacuum instability is essential already in laboratory conditions, almost any electric-like external field is supercritica $1^{1}$ and the particle creation effect turns out to be dominant (under certain conditions) in various quantum processes in the external electric-like field in the graphene. Application of QED with strong field and unstable vacuum to describing quantum processes in graphene requires nonperturbative methods. In particular, the conductivity of graphene, especially in the so-called nonlinear regime, was studied in Ref. [4] using such methods. The production of electron-hole pairs was recently observed in graphene while investigating a behavior of its current-voltage characteristics [5].

It is known that one of the graphene property is its great sensitivity to impurities and defects, which is associated with the vanishing density of states of current carriers. The transport of electrons in the presence of charged impurities such as Coulomb centers becomes one of the most important topics relating to achieving maximum carrier mobility in graphene [6]. Because the Coulomb law remains unchanged in undoped graphene and is approximately retained for small and moderate doping, the scattering processes are essentially controlled by an unscreened Coulomb potential, unlike ordinary metals where the screening is complete [7].

A potential induced by substrate can break the symmetry of the honeycomb lattice and generate a gap in the graphene electron spectrum. The gap then suggests that the motion of electrons can be described by the $2+1$ massive Dirac equations. The problem of the electron spectra of gapped graphene with Coulomb impurities was considered in [8, 9, 10, 11].

Relativistic scattering theory for $2+1$ Dirac electrons in graphene by Coulomb impurities uses solutions of spectral problem for a Dirac Hamiltonian in $2+1$ dimensions with the corresponding point-like or regularized three-dimensional Coulomb potential. The corresponding spectral problem in $3+1$ dimensions was discussed in detail in Refs. [12, 13, 14, 15] for the point-like Coulomb potential and in Refs. [15, 16] for a regularized Coulomb potential. For the point-like Coulomb potential a consistent treatment of this problem depends essentially on a correct definition of the Dirac Hamiltonian as a self-adjoint (s.a. in what follows) operator in an appropriate Hilbert space. Whereas in $3+1$ dimensions peculiarities of such a definition apppear only for nuclei with large $Z>119$, which do not exist in laboratory conditions, for Coulomb impurities in the graphene this threshold is much less due to properties of Dirac excitations in the graphene.

In this paper, we consider the problems of correctly defining the Dirac Hamiltonian for quasiparticles in gapped graphene in the presence of a Coulomb impurity of a charge $Z$ as the s.a. operator and of its spectral analysis. We present a rigorous consideration of all aspects of these problems based on theory of s.a. extensions of symmetric operators [13]. We also study the spectral problem in the case of a regularized Coulomb field of impurities, which allows an exact analytical formulation.

The paper is organized as follows. We begin with introducing a basic information and equations explaining

\footnotetext{
1 Note also that the standard QED vacuum in $(3+1)$ dimensions becomes unstable due to the Coulomb attraction between electron and positron above a critical value of the fine-structure constant [2, 3], $\alpha_{c r}=\pi / 8$ or, with its genuine value of $\alpha=1 / 137$, but if an external magnetic field above $10^{42} \mathrm{G}$ is imposed [3].
} 
the setting of the problem, Section 2. In Section 3 , we describe a mathematically rigorous procedure for reducing the problem of constructing s.a. rotationally invariant Dirac Hamiltonian in the full Hilbert space to the problem of constructing s.a. one-dimensional partial radial Hamiltonians with certain angular momenta. The further consideration is divided into two parts. In the first part (Sections 4 6), we consider the point-like Coulomb field of impurities in gapped graphene. In Section 4, we construct a general solution of radial equations, as well as some particular solutions of these equations used in what follows. Section 5 is devoted to constructing s.a. partial radial Hamiltonians with arbitrary admissible angular momentum $j$ and to solving the corresponding spectral problems in four different regions of the upper $(j, Z)$ half-plane, the regions differ by a character .of s.a. boundary conditions at the origin specifying partial radial Hamiltonians. Section 6 is devoted to desribing pecularities of the total Hamiltonian in dependence of a charge $Z$. The second part of the paper (Sections 7 10 deals with a regularized Coulomb field of impurities in the gapped graphene. In the case of a specially regularized Coulomb field, we deduce the exact equations which allow finding the point spectrum (located in the semi-interval $[-m, m))$ together with the corresponding bound states, Section 7. In the Section 9, we present the exact equations for charges that give bound states with energy $E=-m$ that coincides with the upper boundary of the negative branch of the continuous spectrum. In the Section 10, we discuss the concept of supercritical impurity charge. Section 11 is devoted to a discussion of our results in the both parts of the paper.

\section{Dirac equation in $2+1$ dimensions with point-like Coulomb field}

Here we will consider the spectrum of quasiparticle excitations in the presence of a single Coulomb impurity of charge $Z e$ placed in the middle of the hexagon of the graphene lattice. More specifically, we restrict ourselves by neighborhoods of the two so called Dirac points which are the regions of the quasiparticle spectrum most sensitive to an influence of the impurity. In addition, we suppose that the impurity is a fully stripped ion, so that $Z$ is an atomic number.

We introduce a Cartesian coordinate system with $O x$ and $O y$ axes lying in the graphene plane and the $O z$ axis normal to the plane. The impurity is situated at the origin. The Coulomb field is weakened by the polarization of the crystal at distances greater than the interatomic ones. To take this effect into account, a macroscopic permittivity $\epsilon$ (which is also called an effective dielectric permittivity) must be introduced into the Coulomb potential, which reads

$$
V(\rho)=-\frac{Z e^{2}}{\epsilon}\left(\frac{1}{\rho}\right), \quad \rho=|\mathbf{r}|=\sqrt{x^{2}+y^{2}},
$$

where $\mathbf{r}=(x, y)$ is a two-dimensional vector (in the graphene plane $z=0$ ).

Quasiparticles in graphene live in two-dimensional space, whereas the Coulomb field created by an impurity lives in three-dimensional space, and therefore the Coulomb potential in the graphene plane $z=0$ behaves like

$\rho^{-1}$. If we were to consider the electric field created by an impurity of charge $q$ that lives in two-dimensional 
space, the electric potential $\Phi$ would be $\Phi=q /(2 \pi \epsilon) \log (1 / \rho)$ as it follows from the Poisson equation $\Delta \Phi=$ $-(q / \epsilon) \delta(\mathbf{r})$ in two-dimensional space.

In the case that the shielding is due to electrons in graphene, the RPA method gives $\epsilon \approx 5$ (see Ref. [17]). If we completely neglect effects of a polarization in the graphene, but the graphene is on a $\mathrm{SiO}_{2} \mathrm{substrate}$ then $\epsilon \approx 2.5$. (see also Ref. [18]).

We note that potentials induced by the substrate can violate the symmetry of the lattice and create gaps in the electronic spectrum: the gaps between the conduction band and the valence band. By choosing a substrate, it is possible to adjust the effective mass of charge carriers and investigate phenomena absent in the massless case. In this paper, we assume that charge carriers in graphene can have some effective mass $m_{*}>0$.

To distinguish the two different Dirac points in the graphene, we introduce a parameter, or an index, $s= \pm 1$, which we will call isospin in the following because of a certain analogy with the latter. We let $\mathbf{K}_{s}$ denote the Dirac points, their coordinates in the Brillouin zone are chosen as $\mathbf{K}_{s}=(4 \pi s /(3 a), 0)$, where $a=2.46 \AA$ is the lattice constant.

As we said above, we restrict our consideration to the neighborhoods of the two Dirac points, which means that we restrict ourselves to low-energy quasiparticle excitations and low-energy quasiparticle transitions. In this approximation, the complete Hilbert space $\mathfrak{H}_{\text {tot }}$ of quantum states of an quasiparticle in graphene is a direct orthogonal sum of two Hilbert spaces $\mathfrak{H}_{s}, s= \pm 1$, each of which is related to the corresponding Dirac point $\mathbf{K}_{s}$, the both Hilbert spaces $\mathfrak{H}_{s}$ are Hilbert spaces of two-dimensional doublets, so that $\mathfrak{H}_{\text {tot }}=\mathfrak{H}_{1} \oplus \mathfrak{H}_{-1}$, $\mathfrak{H}_{1}=\mathfrak{H}_{-1}=\mathfrak{H}=L^{2}\left(\mathbb{R}^{2}\right) \oplus L^{2}\left(\mathbb{R}^{2}\right)$.

Usually, in the graphene, intervalley processes are not taken into account referring to the long-range nature of the Coulomb field. Formally, this means that transitions between the Hilbert spaces $\mathfrak{H}_{s}$ are not considered, such that the total quasiparticle Hamiltonian $\hat{H}_{\text {tot }}$ is a direct orthogonal sum of two Hamiltonians $\widehat{\mathcal{H}}_{s}, s= \pm 1$, each acting in the respective Hilbert space $\mathfrak{H}_{s}$ and can be treated independently.

In the framework of the tight-binding approximation and for low quasiparticle energy, the stationary Schrödinger equation for an electron reduces to the following two independent equations for the envelopes of Bloch functions in the neighborhood of each Dirac point $\mathbf{K}_{s}$ [19]:

$$
\check{\mathcal{H}}_{s} \Psi_{s}=\mathcal{E} \Psi_{s}, \quad s= \pm 1
$$

where wave functions $\Psi_{s}$ are doublets depending on $\mathbf{r}, \Psi_{s}=\Psi_{s}(\mathbf{r})=\left\{\psi_{s \alpha}(\mathbf{r}), \alpha=1,2\right\}$, whose components $\psi_{s \alpha}(\mathbf{r})$ are the envelopes of the Bloch functions in two respective sublattices $A$ and $B$, and $\check{\mathcal{H}}_{s}$ are the corresponding Dirac differential operations:

$$
\begin{gathered}
\check{\mathcal{H}}_{s}=\hbar v_{F}\left(-i\left[s \sigma_{x} \partial_{x}+\sigma_{y} \partial_{y}\right]-\frac{g}{\rho}\right)+\frac{\Delta_{d o p}}{2} \sigma_{z}, \\
g=\frac{1}{\hbar v_{F}} \frac{Z e^{2}}{\epsilon}=\alpha_{F} \frac{Z}{\epsilon}=\alpha_{F} Z_{e f f}, \quad Z_{e f f}=\frac{Z}{\epsilon},
\end{gathered}
$$


where $v_{F} \approx 10^{6} \mathrm{~cm} / \mathrm{s}$ is the Fermi velocity, $\Delta_{d o p}=2 m_{*} v_{F}^{2}$ is the energy gap, $\alpha_{F}=e^{2} /\left(\hbar v_{F}\right)$ is the graphene "fine structure constant", and $\left\{\sigma_{x}, \sigma_{y}, \sigma_{z}\right\}$ are the Pauli matrices.

In what follows, we use the following notation: $\check{H}_{s}=\left(\hbar \nu_{F}\right)^{-1} \check{\mathcal{H}}_{s}, E=\left(\hbar \nu_{F}\right)^{-1} \mathcal{E}$ and $m=\left(2 \hbar v_{F}\right)^{-1} \Delta_{\text {dop }}$. Note that the variable $E$ and the parameter $m$ are of dimension of the inverse length. Equation (1) then reads

$$
\check{H}_{s} \Psi_{s}(\mathbf{r})=E \Psi_{s}(\mathbf{r}), \quad s= \pm 1
$$

where the differential operations $\check{H}_{s}$ in the Cartesian and in the polar coordinates $\rho, \phi,(x=\rho \cos \phi, y=\rho \sin \phi)$ have the respective forms:

$$
\begin{aligned}
\check{H}_{s} & =-i\left(s \sigma_{x} \partial_{x}+\sigma_{y} \partial_{y}\right)-\frac{g}{\rho}+m \sigma_{z} \\
& =-i\left(s \cos \phi \sigma_{x}+\sin \phi \sigma_{y}\right) \frac{\partial}{\partial \rho}+\frac{i}{\rho}\left(s \sin \phi \sigma_{x}-\cos \phi \sigma_{y}\right) \frac{\partial}{\partial \phi}-\frac{g}{\rho}+m \sigma_{z}
\end{aligned}
$$

To assign a specific meaning of a quantum-mechanical eigenvalue problem for certain Hamiltonians $\hat{H}_{s}$ to Eqs. (3), we have to solve the two problems. The first one is to define, or construct, the Hamiltonians $\hat{H}_{s}$ as s.a. operators with certain domains in the Hilbert space $\mathfrak{H}=L^{2}\left(\mathbb{R}^{2}\right) \oplus L^{2}\left(\mathbb{R}^{2}\right)$ of doublet functions acting on their domains by the respective differential operations $\check{H}_{s}$ (4) (for brevity, we will say that the operators $\hat{H}_{s}$ are associated with differential operations $\check{H}_{s}$ ). The second problem is to perform the spectral analysis of the obtained Hamiltonians, i.e., to evaluate their spectra and the corresponding (generally generalized) eigenfunctions. In solving the both problems, we follow the way adopted in Refs. [12] in the case of the 3-dimensional Dirac equation with point-like and regularized Coulomb fields.

\section{Reduction to radial problem}

We begin with defining an initial symmetric operators $\hat{H}_{s}^{i n}$ in the Hilbert space $\mathfrak{H}=L^{2}\left(\mathbb{R}^{2}\right) \oplus L^{2}\left(\mathbb{R}^{2}\right)$ associated with the respective differential operations $\check{H}_{s}$ (4). Because coefficient functions of differential operations $\check{H}_{s}$ are smooth outside the origin, we choose the space of smooth compactly supported doublets for the domains $D\left(\hat{H}_{s}^{i n}\right)$ of $\hat{H}_{s}^{i n}$.

Thus, one can avoid troubles with a behavior of doublets at infinity. To avoid troubles with the $1 / \rho$ singularity of the Coulomb potential at the origin, we additionally require that all doublets in $D\left(\hat{H}_{s}^{i n}\right)$ vanish in some neighborhood of the origin, specific to each doublet. Note that the domains $D\left(\hat{H}_{s}^{i n}\right)$ (which are the same for the both values $s$ ) are dense in $\mathfrak{H}$. The operators $\hat{H}_{s}^{\text {in }}$ are thus defined as

$$
\hat{H}_{s}^{i n}=\left\{\begin{array}{l}
D\left(\hat{H}_{s}^{i n}\right)=\left\{\Psi(\mathbf{r}): \psi_{\alpha}(\mathbf{r}) \in C_{0}^{\infty}\left(\mathbb{R}^{2} \backslash\{0\}\right)\right\} \\
\hat{H}_{s}^{i n} \Psi(\mathbf{r})=\check{H}_{s} \Psi(\mathbf{r}) .
\end{array}\right.
$$

The symmetricity of $\hat{H}_{s}^{i n}$ is evident. 
We construct s.a. Hamiltonians $\hat{H}_{s}$ as s.a. extensions of the respective initial symmetric operators $\hat{H}_{s}^{i n}$, to emphasize this circumstance, we introduce an additional index $\mathfrak{e}$ to $\hat{H}_{s}, \hat{H}_{s} \rightarrow \hat{H}_{s}^{\mathfrak{e}}$. There exist different s.a. extensions of a given $\hat{H}_{s}^{i n}$, such that the index $\mathfrak{e}$ will be replaced by a more informative index in what follows.

We require that $\hat{H}_{s}^{\mathfrak{e}}$ be rotationally invariant as well as the initial symmetric operators $\hat{H}_{s}^{i n}$ are. The meaning of this requirement is explained below.

There are the two different unitary representations $U_{s}$ of the rotation group $\operatorname{Spin}(2)$ in $\mathfrak{H}$ which are connected with the respective operators $\hat{H}_{s}^{i n}$. The generator $\hat{J}_{s}$ of the representation $U_{s}$, conventionally called the angular momentum operator (there are two of them), is a s.a. operator in $\mathfrak{H}$ defined on absolutely continuous and periodic in $\phi \in[0,2 \pi]$ doublets and associated with the differential operation $\check{J}_{s}=-i \partial / \partial \phi+s \sigma_{z} / 2$. For each $s$, the Hilbert space $\mathfrak{H}$ is represented as a direct orthogonal sum

$$
\mathfrak{H}=\sum_{j}^{\oplus} \mathfrak{H}_{s j}, \quad j= \pm 1 / 2, \pm 3 / 2 \ldots
$$

of subspaces $\mathfrak{H}_{s j}$ that are the eigenspaces of the angular momentum operator $\hat{J}_{s}$ corresponding to all its eigenvalues $j= \pm 1 / 2, \pm 3 / 2 \ldots$ The subspace $\mathfrak{H}_{s j}$ with given $s, j$ consists of doublets $\Psi_{s j}$ of the form

$$
\Psi_{s j}(\mathbf{r})=\frac{1}{\sqrt{2 \pi \rho}} e^{i j \phi}\left(\begin{array}{c}
e^{-i s \phi / 2} f(\rho) \\
-i s e^{+i s \phi / 2} g(\rho)
\end{array}\right) \in \mathfrak{H}_{s j},
$$

they are the eigenfunctions of $\hat{J}_{s}$ with the eigenvalue $j, \hat{J}_{s} \Psi_{s j}(\mathbf{r})=\check{J}_{s} \Psi_{s j}(\mathbf{r})=j \Psi_{s j}(\mathbf{r})$. We note that spectra of both operators $\hat{J}_{-1}$ and $\hat{J}_{1}$ are the same. The functions $f(\rho)$ and $g(\rho)$ are called the radial functions. In the physical language, decompositions (5) and (6) correspond to the expansion of doublets $\Psi(\mathbf{r}) \in \mathfrak{H}$ in terms of eigenfunctions of the two different angular momentum operators $\hat{J}_{-1}$ and $\hat{J}_{1}$.

The following remark is very useful in our constructions. Let $\mathbb{L}^{2}\left(\mathbb{R}_{+}\right)$be the Hilbert space of radial doublets,

$$
F(\rho)=\left(\begin{array}{c}
f(\rho) \\
g(\rho)
\end{array}\right) \in \mathbb{L}^{2}\left(\mathbb{R}_{+}\right)
$$

with the scalar product

$$
\left(F_{1}, F_{2}\right)=\int_{0}^{\infty} F_{1}{ }^{+}(\rho) F_{2}(\rho) d \rho=\int_{0}^{\infty}\left[\overline{f_{1}(\rho)} f_{2}(\rho)+\overline{g_{1}(\rho)} g_{2}(\rho)\right] d \rho,
$$

so that $\mathbb{L}^{2}\left(\mathbb{R}_{+}\right)=L^{2}\left(\mathbb{R}_{+}\right) \oplus L^{2}\left(\mathbb{R}_{+}\right)$. Then Eq. 6) and the relation

$$
\left\|\Psi_{s j}\right\|^{2}=\int_{0}^{\infty}\left[|f(\rho)|^{2}+|g(\rho)|^{2}\right] d \rho
$$

show that each subspace $\mathfrak{H}_{s j} \subset \mathfrak{H}$ is unitarily equivalent to $\mathbb{L}^{2}\left(\mathbb{R}_{+}\right)$,

$$
\Psi_{s j}(\mathbf{r})=V_{s j} F(\rho), \quad F(\rho)=V_{s j}^{-1} \Psi_{s j}(\mathbf{r}) .
$$


If necessary, an explicit form of operators $V_{s j}$ and $V_{s j}^{-1}$ can be easily written down.

The initial symmetric operators $\hat{H}_{s}^{i n}$ are rotationally invariant. Namely, each $\hat{H}_{s}^{i n}$ is invariant from the standpoint of the representation $U_{s}$ of the rotation group. By definition, this means that each subspace $\mathfrak{H}_{s j}$ (the eigenspace of the generator $\hat{J}_{s}$ with eigenvalue $j$ ) reduces the operator $\hat{H}_{s}^{i n}$. In other words, the operator $\hat{H}_{s}^{i n}$ commutes with orthoprojectors $P_{s j}$ on the subspaces $\mathfrak{H}_{s j}$, see [20]. This means the following. Let $\Psi_{s}(\mathbf{r})=$ $\sum_{j} \Psi_{s j}(\mathbf{r}) \in D\left(\hat{H}_{s}^{i n}\right)$. Then

$$
\Psi_{s j}=P_{s j} \Psi_{s} \in D\left(\hat{H}_{s}^{i n}\right), \quad \hat{H}_{s}^{i n} \Psi_{s}=\sum_{j} \hat{H}_{s j}^{i n} \Psi_{s j}
$$

where $\hat{H}_{s j}^{i n}=P_{s j} \hat{H}_{s}^{i n} P_{s j}=\hat{H}_{s}^{i n} P_{s j}$ are the so called parts of $\hat{H}_{s}^{i n}$ lying in $\mathfrak{H}_{s j}$, their rule of acting is given by the certain first-order differential operation in $\rho$, which is easily evaluated, we will see it below. Thus, each initial symmetric operator $\hat{H}_{s}^{i n}$ is a direct orthogonal sum of its parts,

$$
\hat{H}_{s}^{i n}=\sum_{j} \oplus \hat{H}_{s j}^{i n},
$$

and a study of the rotationally invariant $\hat{H}_{s}^{i n}$ is reduced to a study of its parts $\hat{H}_{s j}^{i n}$. Note that these facts are essentially based on the formal commutativity of the differential operations $\check{H}_{s}$ and $\check{J}_{s},\left[\check{H}_{s}, \check{J}_{s}\right]=0$.

We would like to make in passing a general note concerning a rather popular understanding of quantummechanical symmetry in physical literature by the above example of rotational symmetry. In physical literature, the statement that the operators $\hat{H}_{s}^{i n}$ are rotationally invariant, and as a consequence, their study is reduced to a study of their parts $\hat{H}_{s j}^{i n}$ acting in $\mathfrak{H}_{s j}$, is usually identified with the statement that the operators $\hat{H}_{s}^{i n}$ commute with the respective generators $\hat{J}_{s}$ of the rotation group, which in turn is often identified with the commutativity of the differential operations $\check{H}_{s}$ and $\check{J}_{s},\left[\hat{H}_{s}^{i n}, \hat{J}_{s}\right]=\left[\check{H}_{s}, \check{J}_{s}\right]=0$. Strictly speaking, such a statement is improper twice: the formal commutativity of differential operations in no way implies the commutativity of the associated operators, the more so as the commutator of two unbounded operators in Hilbert space are generally not defined.

Each $\hat{H}_{s j}^{i n}$ is a symmetric operator in the subspace $\mathfrak{H}_{s j}$. It evidently induces a symmetric operator $\hat{h}_{i n}(Z, j, s)$ in the Hilbert space $\mathbb{L}^{2}\left(\mathbb{R}_{+}\right)$that is unitarily equivalent to $\hat{H}_{s j}^{i n}, \hat{h}_{i n}(Z, j, s) \stackrel{\text { def }}{=} V_{s j}^{-1} \hat{H}_{s j}^{i n} V_{s j}$, so that $\hat{h}_{i n}(Z, j, s) F=$ $V_{s j}^{-1} \hat{H}_{s j}^{i n} \Psi_{s j}, \Psi_{s j}=V_{s j} F$. The $\hat{h}_{i n}(Z, j, s)$ is given by

$$
\hat{h}_{i n}(Z, j, s)=\left\{\begin{array}{l}
D_{h_{i n}(Z, j, s)}=\mathbb{C}_{0}^{\infty}\left(\mathbb{R}_{+}\right), \\
\hat{h}_{i n}(Z, j, s) F(\rho)=\check{h}(Z, j, s) F(\rho),
\end{array}\right.
$$

where $\mathbb{C}_{0}^{\infty}\left(\mathbb{R}_{+}\right)=C_{0}^{\infty}\left(\mathbb{R}_{+}\right) \oplus C_{0}^{\infty}\left(\mathbb{R}_{+}\right)$and the differential operation $\breve{h}(Z, j, s)$ reads

$$
\begin{aligned}
& \check{h}(Z, j, s)=-i \sigma_{y} \frac{d}{d \rho}+\frac{\kappa}{\rho} \sigma_{x}-\frac{g}{\rho}+m \sigma_{z}, \\
& \kappa=-s j, \quad g=\alpha_{F} \epsilon^{-1} Z,
\end{aligned}
$$

we call it the partial radial differential operation. 
Constructing s.a. rotationally invariant Hamiltonians $\hat{H}_{s}$ as s.a. extensions of the initial symmetric operators $\hat{H}_{s}^{i n}, \hat{H}_{s}=\hat{H}_{s}^{\mathfrak{e}}$, reduces to constructing s.a. partial radial Hamiltonians $\hat{h}(Z, j, s)$ in $\mathbb{L}^{2}\left(\mathbb{R}_{+}\right)$as s.a. extensions of the initial symmetric partial radial operators $\hat{h}_{i n}(Z, j, s), \hat{h}(Z, j, s)=\hat{h}_{\mathfrak{e}}(Z, j, s)$. This goes as follows. Let $\hat{h}_{\mathfrak{e}}(Z, j, s)$ be such extensions, they evidently induce the s.a. extensions $\hat{H}_{s j}^{\mathfrak{e}}=V_{s j} \hat{h}_{\mathfrak{e}}(Z, j, s) V_{s j}^{-1}$ of the initial symmetric operators $\hat{H}_{s j}^{i n}$ in the subspaces $\mathfrak{H}_{s j}$. Then the direct orthogonal sum of the partial operators $\hat{H}_{s j}^{\mathfrak{e}}$,

$$
\hat{H}_{s}^{\mathfrak{e}}=\sum_{j}^{\oplus} \hat{H}_{s j}^{\mathfrak{e}}
$$

is a s.a. rotationally invariant extension of the initial symmetric operator $\hat{H}_{s}^{\text {in }}$, the desired s.a. rotationally invariant Hamiltonian $\hat{H}_{s}=\hat{H}_{s}^{\mathfrak{e}}$ in $\mathfrak{H}$. Conversely, any s.a. rotationally invariant extension of the initial symmetric operator $\hat{H}_{s}^{i n}$ has structure 10. The spectrum of the Hamiltonian $\hat{H}_{s}^{\mathfrak{e}}$ is a union of the spectra of partial radial Hamiltonians, spec $\hat{H}_{s}^{\mathfrak{e}}=\cup_{j}$ spec $\hat{h}_{\mathfrak{e}}(Z, j, s)$, and the corresponding eigenfunctions related to $\mathfrak{H}_{s j}$ are obtained from the eigenfunctions of $\hat{h}_{\mathfrak{e}}(Z, j, s)$ in $\mathbb{L}^{2}\left(\mathbb{R}_{+}\right)$by the transformation $V_{s j}$, see (7).

We already said above that in our consideration, we follow a similar consideration for the s.a. rotationally invariant (with respect to the Spin(3) group) Dirac Hamiltonian for an electron in the Coulomb field in three dimensions [12, 13, 14, 16]. We recall that in [12, 13, 14, 16, there was solved the problem of constructing and spectral analysis of s.a. partial radial Hamiltonians $\hat{h}_{\mathfrak{e}}(Z, j, \zeta)$ in $\mathbb{L}^{2}\left(\mathbb{R}_{+}\right)$as s.a. extensions of the initial partial radial symmetric operators $\hat{h}_{i n}(Z, j, \zeta)$ defined on $\mathbb{C}_{0}^{\infty}\left(\mathbb{R}_{+}\right)$and associated with the differential operations

$$
\check{h}(Z, j, \zeta)=-i \sigma_{y} \frac{d}{d r}+\frac{\varkappa}{r} \sigma_{x}-\frac{q}{r}+m \sigma_{z},
$$

where $r=\sqrt{x^{2}+y^{2}+z^{2}}, \varkappa=\zeta j, j=1 / 2,3 / 2, \ldots$ is the 3 -dimensional angular momentum quantum number, $\zeta= \pm 1$ is the spin number, $q=\alpha Z, \alpha$ is the fine structure constant, $m$ is the electron mass. The differential operation 111 differs from differential operation $\breve{h}(Z, j, s)$ given by Eq. 99 only by values and an interpretation of parameters involved. To make a comparison with the three-dimensional problem, it is convenient to introduce the parameter $\zeta=\zeta(j, s)=-s \operatorname{sgn}(j)= \pm 1, \kappa=\zeta(j, s)|j|$.

\section{General solution of radial equations}

We now turn to the general solution of the system of two linear ordinary differential equations

$$
\check{h}(Z, j, s) F(\rho)=W F(\rho), \quad F(\rho)=\left(\begin{array}{l}
f(\rho) \\
g(\rho)
\end{array}\right), \quad W \in \mathbb{C},
$$

which is necessary in future in evaluating spectra and eigenfunctions of partial radial Hamiltonians; the system (12) is sometimes called the (stationary) partial radial Schrödinger equation. Real values of $W$ are denoted by $E$ in what follows. For our purposes, it is actually sufficient to consider $W$ belonging to the upper complex half-plane, $W=E+i y, y \geq 0$. What is more, the limit $W \rightarrow E+i 0$, is of our main interest. 
System 12 in terms of $f(\rho)$ and $g(\rho)$ has the form:

$$
\begin{aligned}
& \frac{d f}{d \rho}+\frac{\kappa}{\rho} f(\rho)-\left(W+m+\frac{g}{\rho}\right) g(\rho)=0 \\
& \frac{d g}{d \rho}-\frac{\kappa}{\rho} g(\rho)+\left(W-m+\frac{g}{\rho}\right) f(\rho)=0 .
\end{aligned}
$$

We call Eqs. (13) the radial equations. The radial equations for the three-dimensional problem have the same form.

For completeness, we present the general solution of the radial equations following the standard procedure [21, 13. We begin with a change of variables,

$$
\begin{aligned}
& f(\rho)=z^{\Upsilon} e^{-z / 2}[Q(z)+P(z)], \\
& g(\rho)=i \Lambda z^{\Upsilon} e^{-z / 2}[Q(z)-P(z)], \quad z=-2 i K \rho,
\end{aligned}
$$

where

$$
\begin{aligned}
& \Upsilon^{2}=\kappa^{2}-g^{2}, \quad W \pm m=r_{ \pm} e^{i \phi_{ \pm}}, \quad 0 \leq \phi_{ \pm} \leq \pi, \quad r_{ \pm} \geq 0 \\
& \Lambda=\sqrt{\frac{W-m}{W+m}}=\sqrt{\frac{r_{-}}{r_{+}}} e^{-i\left(\phi_{+}-\phi_{-}\right) / 2}, \quad K=\sqrt{W^{2}-m^{2}}=\sqrt{r_{+} r_{-}} e^{i\left(\phi_{+}+\phi_{-}\right) / 2} .
\end{aligned}
$$

In new variables, the system of radial equations $[13$ reads

$$
\begin{aligned}
& z \frac{d^{2} Q(z)}{d z^{2}}+(\beta-z) \frac{d Q(z)}{d z}-\alpha Q(z)=0, \quad P(z)=-\frac{1}{b_{+}}\left(z \frac{d}{d z}+\alpha\right) Q(z), \\
& \beta=1+2 \Upsilon, \quad \alpha=\alpha_{+}, \quad \alpha_{+}=\Upsilon+\frac{g W}{i K}, \quad b_{+}=\kappa+\frac{g m}{i K} .
\end{aligned}
$$

The equation for the function $Q(z)$ is the well-known confluent hypergeometric equation.

Let $\Upsilon \neq-n / 2, n \in \mathbb{N}$. The general solution of the confluent hypergeometric equation is then a linear combination of the standard confluent hypergeometric functions $\Phi(\alpha, \beta ; z)$ and $\Psi(\alpha, \beta ; z)$,

$$
Q(z)=A \Phi(\alpha, \beta ; z)+B \Psi(\alpha, \beta ; z)
$$

where $A, B=$ const,

$$
\Psi(\alpha, \beta ; z)=\frac{\Gamma(1-\beta)}{\Gamma(\alpha-\beta+1)} \Phi(\alpha, \beta ; z)+\frac{\Gamma(\beta-1)}{\Gamma(\alpha)} z^{1-\beta} \Phi(\alpha-\beta+1,2-\beta ; z) .
$$

Then using the relations

$$
\begin{aligned}
& \left(z \frac{d}{d z}+\alpha\right) \Phi(\alpha, \beta ; z)=\alpha \Phi(\alpha+1, \beta ; z), \\
& \left(z \frac{d}{d z}+\alpha\right) \Psi(\alpha, \beta ; z)=\alpha(\alpha-\beta+1) \Psi(\alpha+1, \beta ; z), \\
& \alpha-\beta+1=-\alpha_{-}, \quad \alpha_{+} \alpha_{-}=b_{+} b_{-}, \quad a=\frac{\alpha_{+}}{b_{+}}, \\
& \alpha_{-}=\Upsilon-\frac{g W}{i K}, \quad b_{-}=\kappa-\frac{g m}{i K},
\end{aligned}
$$


we find that the general solution of system (14) is given by

$$
\begin{aligned}
Q(z) & =A \Phi(\alpha, \beta ; z)+B \Psi(\alpha, \beta ; z) \\
P(z) & =-A a \Phi(\alpha+1, \beta ; z)+B b_{-} \Psi(\alpha+1, \beta ; z) .
\end{aligned}
$$

And finally, using the relations

$$
\Phi(\alpha+1, \beta ; z)=e^{z} \Phi(\beta-\alpha-1, \beta ;-z), \quad i \Lambda \frac{1+a}{1-a}=\frac{\kappa+\Upsilon}{g},
$$

we represent the general solution of radial equations $(13)$ in the following form:

$$
\begin{aligned}
& F=A X(\rho, \Upsilon, W)+B z^{\Upsilon} e^{-z / 2}\left[\Psi(\alpha, \beta ; z) \varrho_{+}-b_{-} \Psi(\alpha+1, \beta ; z) \varrho_{-}\right] \\
& \varrho_{ \pm}=( \pm 1, i \Lambda)^{T},
\end{aligned}
$$

where the doublet $X(\rho, \Upsilon, W)$ is

$$
\begin{gathered}
X(\rho, \Upsilon, W)=\frac{(-2 i K / m)^{-\Upsilon}}{1-a} z^{\Upsilon} e^{-z / 2}\left[\Phi(\alpha, \beta ; z) \varrho_{+}+a \Phi(\alpha+1, \beta ; z) \varrho_{-}\right] \\
=\frac{(m \rho)^{\Upsilon}}{2}\left[\Phi_{+}(\rho, \Upsilon, W)+\Phi_{-}(\rho, \Upsilon, W) \Xi\right] d_{+}, \\
\Phi_{+}(\rho, \Upsilon, W)=e^{i K \rho} \Phi(\alpha, 1+2 \Upsilon,-2 i K \rho)+e^{-i K \rho} \Phi\left(\alpha_{-}, 1+2 \Upsilon, 2 i K \rho\right), \\
\Phi_{-}(\rho, \Upsilon, W)=\frac{1}{i K}\left[e^{i K \rho} \Phi(\alpha, 1+2 \Upsilon,-2 i K \rho)-e^{-i K \rho} \Phi\left(\alpha_{-}, 1+2 \Upsilon, 2 i K \rho\right)\right], \\
\Xi=\left(\begin{array}{cc}
0 & m+W \\
m-W & 0
\end{array}\right), d_{ \pm}=\left(1, \frac{\kappa \pm \Upsilon}{g}\right)^{T} .
\end{gathered}
$$

In what follows, we use some particular solutions of radial equations 13 corresponding to a specific choice of the constants $A$ and $B$ and the parameter $\Upsilon$. First, we introduce a new quantity $\Upsilon_{+}$as follows:

$$
\Upsilon_{+}=\Upsilon_{+}(g, j)= \begin{cases}\gamma=\sqrt{\kappa^{2}-g^{2}} \geq 0, & g \leq|\kappa|, \\ i \sigma=i \sqrt{g^{2}-\kappa^{2}}, \quad \sigma>0, & g>|\kappa| .\end{cases}
$$

This quantity $\Upsilon_{+}$as a function of $g$ has zeros at the points $g=g_{\mathrm{c}}(j)=|\kappa|=|j|$.

In the case $\Upsilon_{+} \neq 0\left(g \neq g_{\mathrm{c}}(j)\right)$, we take two linearly independent solutions $F_{1}$ and $F_{2}$ forming a fundamental system of solutions of system 13 ,

$$
\begin{aligned}
& F_{1}(\rho ; W)=X\left(\rho, \Upsilon_{+}, W\right)=(m \rho)^{\Upsilon_{+}} d_{+}+O\left(\rho^{\Upsilon_{+}+1}\right), \quad \rho \rightarrow 0, \\
& F_{2}(\rho ; W)=X\left(\rho,-\Upsilon_{+}, W\right)=(m \rho)^{-\Upsilon_{+}} d_{-}+O\left(\rho^{-\Upsilon_{+}+1}\right), \quad \rho \rightarrow 0,
\end{aligned}
$$

it is remarkable that the both doublets $F_{1}$ and $F_{2}$ are real entire in $W$. Their Wronskian is $\operatorname{Wr}\left(F_{1}, F_{2}\right)=$ $-2 \Upsilon_{+} g^{-1}$. If $\operatorname{Im} W>0$ and $\rho \rightarrow \infty$, the both doublets $F_{1}(\rho ; W)$ and $F_{2}(\rho ; W)$ increase exponentially. 
Another useful solution $F_{3}$ is given by (15) with $A=0, \Upsilon=\Upsilon_{+}$and a special choice for $B=B(W)$,

$$
\begin{gathered}
F_{3}(\rho ; W)=B(W) z^{\Upsilon} e^{-z / 2}\left[\Psi(\alpha, \beta ; z) \varrho_{+}-b_{-} \Psi(\alpha+1, \beta ; z) \varrho_{-}\right] \\
=\Gamma\left(-2 \Upsilon_{+}\right) F_{1}(\rho ; W)-\frac{\omega(W) F_{2}(\rho ; W)}{\operatorname{Wr}\left(F_{1}, F_{2}\right)} \\
\omega(W)=\frac{\Gamma\left(1+2 \Upsilon_{+}\right) \Gamma\left(-\alpha_{-}\right)\left[i g K+\left(\kappa+\Upsilon_{+}\right)(W+m)\right]}{g \Gamma(\alpha)\left[i g K+\left(\kappa-\Upsilon_{+}\right)(W+m)\right]}\left(2 e^{-i \pi / 2} \frac{K}{m}\right)^{-2 \Upsilon_{+}}=-\operatorname{Wr}\left(F_{1}, F_{3}\right), \\
B(W)=\frac{1}{2} \Gamma\left(-\alpha_{-}\right)\left[1+\frac{(m+W)\left(\kappa+\Upsilon_{+}\right)}{i g K}\right]\left(2 e^{-i \pi / 2} \frac{K}{m}\right)^{-\Upsilon_{+}} .
\end{gathered}
$$

If $\operatorname{ImW}>0$ and $\rho \rightarrow \infty$, the doublet $F_{3}(\rho ; W)$ decreases exponentially (with a polynomial accuracy).

In the special case of $\Upsilon_{+}=\gamma=0\left(g=g_{\mathrm{c}}(j)\right)$ where the doublets $F_{1}$ and $F_{2}$ coincide, we will consider two linearly independent solutions $F_{1}^{(0)}$ and $F_{2}^{(0)}$ and their linear combination $F_{3}^{(0)}$,

$$
\begin{aligned}
& F_{1}^{(0)}(\rho ; W)=\left.F_{1}(\rho ; W)\right|_{\gamma=0}=\left.d_{+}\right|_{\gamma=0}+O(\rho),\left.\quad d_{+}\right|_{\gamma=0}=(1, \zeta(j, s))^{T}, \rho \rightarrow 0, \\
& F_{2}^{(0)}(\rho ; W)=\left.\partial_{\gamma} F_{1}(\rho ; W)\right|_{\gamma=0}-\frac{\zeta(j, s)}{g_{c}(j)} F_{1}^{(0)}(\rho ; W)=d_{0}(\rho)+O(\rho \log \rho), \rho \rightarrow 0, \\
& d_{0}(\rho)=\left(\log (m \rho)-\zeta(j, s) g_{c}^{-1}(j), \zeta(j, s) \log (m \rho)\right)^{T}, \\
& F_{3}^{(0)}(\rho ; W)=-\lim _{\gamma \rightarrow 0} F_{3}(\rho ; W)=F_{2}^{(0)}(\rho ; W)+f(W) F_{1}^{(0)}(\rho ; W), F_{3}^{(0)} \in \mathbb{L}^{2}\left(\mathbb{R}_{+}\right), \\
& f(W)=g_{c}(j) \omega^{(0)}(W)=\log \left(2 e^{-i \pi / 2} K / m\right)+\psi\left(-i g_{c}(j) W K^{-1}\right) \\
& \quad+(\zeta(j, s)(W-m)+i K))\left(2 g_{c}(j) W\right)^{-1}-2 \psi(1), \quad \psi(x)=\Gamma^{\prime}(x) / \Gamma(x) .
\end{aligned}
$$

The corresponding Wronskians are

$$
\mathrm{Wr}\left(F_{1}^{(0)}, F_{2}^{(0)}\right)=g_{\mathrm{c}}^{-1}(j), \quad \operatorname{Wr}\left(F_{2}^{(0)}, F_{3}^{(0)}\right)=-\omega^{(0)},
$$

where $\omega^{(0)}=\omega^{(0)}(W)$.

\section{Self-adjoint radial Hamiltonians}

Here it is useful to recall what was said in the end of Sec. 3. Because radial differential operation $\check{h}(Z, j, s)$ (9) coincides with radial differential operation $\check{h}(Z, j, \zeta)$ 11, arising in solving the 3-dimensional Coulomb problem in [13], up to the replacement and reinterpretation of the parameters $\kappa \rightarrow \varkappa, g \rightarrow q$, we can use some results in Ref. [12, 13, 14, 16] concerning s.a. partial radial Hamiltonians $\hat{h}_{\mathfrak{e}}(Z, j, \zeta)$ in $\mathbb{L}^{2}\left(\mathbb{R}_{+}\right)$for defining and the spectral analysis of s.a. partial radial Hamiltonians $\hat{h}_{\mathfrak{e}}(Z, j, s)$ in $\mathbb{L}^{2}\left(\mathbb{R}_{+}\right)$under the replacement and an appropriate reinterpretation of the parameters $\varkappa \rightarrow \kappa, q \rightarrow g$.

Because all possible s.a. partial radial Hamiltonians $\hat{h}_{\mathfrak{e}}(Z, j, s)$ are associated with the common differential operation $\breve{h}(Z, j, s) 9$, see just below, their definition reduces to specifying their domains $D_{h(Z, j, s)} \subset \mathbb{L}^{2}\left(\mathbb{R}_{+}\right)$. 
Each operator $\hat{h}_{\mathfrak{e}}(Z, j, s)$ is a s.a. extension of initial symmetric operator $\hat{h}_{\mathrm{in}}(Z, j, s)$ (8) associated with the differential operation $\breve{h}(Z, j, s)\left[9\right.$, s.a. in the sense of Lagrange, and defined on the space $\mathbb{C}_{0}^{\infty}\left(\mathbb{R}_{+}\right)$of smooth compactly supported doublets on the semiaxis $\mathbb{R}_{+}$. Simultaneously, each $\hat{h}_{\mathfrak{e}}(Z, j, s)$ is a s.a. restriction, maybe trivial, of the operator $\hat{h}_{\text {in }}^{+}(Z, j, s)$, the adjoint of $\hat{h}_{\text {in }}(Z, j, s)$, which is associated with the same differential operation $\check{h}(Z, j, s)$, (it is just the reason of that each $\hat{h}_{\mathfrak{e}}(Z, j, s)$ is associated with one and the same differential operation $\breve{h}(Z, j, s))$, and is defined on the so-called natural domain $D_{\breve{h}(Z, j, s)}^{*}\left(\mathbb{R}_{+}\right)$for $\breve{h}(Z, j, s)$ consisting of the doublets $F(\rho) \in \mathbb{L}^{2}\left(\mathbb{R}_{+}\right)$absolutely continuous in $\mathbb{R}_{+}$and such that $\check{h}(Z, j, s) F(\rho) \in \mathbb{L}^{2}\left(\mathbb{R}_{+}\right), D_{h_{\text {in }}(Z, j, s)} \subset$

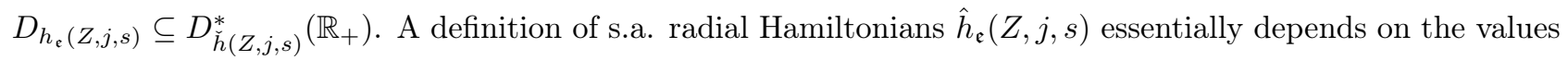
of the parameters $Z$ and $j$, more specifically, on the variable $\Upsilon_{+} 17$.

By definition, the variable $j$ is half-integer-valued, both positive and negative, $j= \pm(n+1 / 2), n \in \mathbb{Z}_{+}$, while the variable $Z$ is nonnegative integer valued, $Z \in \mathbb{Z}_{+}$, so that we deal with the lattice of physically meaningful values of these variables in the upper $(j, Z)$ half-plane. However, it seems convenient to consider the variable $Z$ continuous lying on the nonnegative vertical semiaxis, $Z \in \mathbb{R}_{+}$, and return to its natural integer values of necessity. There are the two regions in the upper $(j, Z)$ half-plane (see Fig. 1 for $\alpha_{F}^{-1} \epsilon=1$ ), we

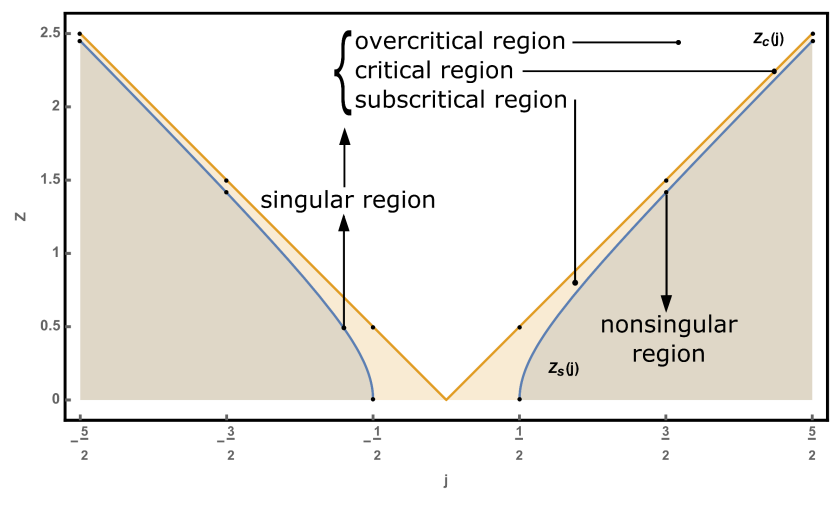

Figure 1: Nonsingular and singular regions in the $(j, Z)$ plane. The singular region consists of subcritical, critical, and overcritical subregions.

call them the nonsingular and singular ones, where the problem of s.a. extensions of $\hat{h}_{\text {in }}(Z, j, s)$ has principally different solutions: in the nonsingular region, s.a. radial Hamiltonians $\hat{h}_{\mathfrak{e}}(Z, j, s)$ are defined uniquely, while in the singular region, they are defined nonuniquely.

These regions are separated by a symmetric singular curve $Z=Z_{\mathrm{s}}(j)$, where

$$
Z_{\mathrm{s}}(j)=\alpha_{F}^{-1} \epsilon \sqrt{j^{2}-\frac{1}{4}}
$$

which is equivalent to $g=g_{\mathrm{s}}(j)=\sqrt{j^{2}-1 / 4}$, or $\Upsilon_{+}=\gamma=1 / 2$, see 17 ; this curve consists of two branches, the right branch begins at the point $(j=1 / 2, Z=0)$, and goes up toward the right approaching a strait-line asymptote $Z=\alpha_{F}^{-1} \epsilon j$, while the left branch begins at the point $(j=-1 / 2, Z=0)$, and goes up toward the 
left approaching a strait-line asymptote $Z=-\alpha_{F}^{-1} \epsilon j$. The nonsingular region (the lower one) is defined by the inequality $Z \leq Z_{\mathrm{s}}(j)$, which is equivalent to the inequality $\Upsilon_{+}=\gamma \geq 1 / 2$, while the singular region (the upper one) is defined by the inequality $Z>Z_{\mathrm{s}}(j)$, which is equivalent to $0 \leq \Upsilon_{+}=\gamma<1 / 2$ or $\Upsilon_{+}=i \sigma, \sigma>0$, see (17).

Note that the singular curve is an upper boundary of the nonsingular region. The value $Z_{\mathbf{s}}(j)$ can be called the maximum nonsingular $Z$-value for a given $j$. For illustration, we present the first $g_{\mathrm{s}}(j)$ values for first small half-integer $j$ :

$$
\begin{aligned}
& g_{\mathrm{s}}( \pm 1 / 2)=0, \quad g_{\mathrm{s}}( \pm 3 / 2)=\sqrt{2}, \\
& g_{\mathrm{s}}( \pm 5 / 2)=\sqrt{6}, \quad g_{\mathrm{s}}( \pm 7 / 2)=2 \sqrt{3} .
\end{aligned}
$$

In what follows, we consider s.a. radial Hamiltonians $\hat{h}(Z, j, s)$ in the nonsingular and singular regions separately.

\subsection{Nonsingular region}

In the nonsingular region, $Z \leq Z_{\mathrm{s}}(j)$, each partial radial Hamiltonian, we let $\hat{h}_{1}(Z, j, s)$ denote it using subscript 1 instead of $\mathfrak{e}$ as the symbol of the nonsingular region (the subscripts 2, 3, 4 together with other relevant indices are used below instead of $\mathfrak{e}$ as symbols of particular subregions of the singular region), is defined uniquely, $\hat{h}_{1}(Z, j, s)=\hat{h}_{\text {in }}^{+}(Z, j, s)$, in other words, the initial symmetric operator $\hat{h}_{\text {in }}(Z, j, s)$ is essentially s.a. because its deficiency indices are $(0,0)$, and the domain of $\hat{h}_{1}(Z, j, s)$ is the natural domain for $\breve{h}(Z, j, s)$, $D_{h_{1}(Z, j, s)}=D_{\breve{h}(Z, j, s)}^{*}\left(\mathbb{R}_{+}\right)$. Functions belonging to $D_{\breve{h}(Z, j, s)}^{*}\left(\mathbb{R}_{+}\right)$have the following asymptotic behavior at the origin and at infinity:

$$
F(\rho)=O\left(\rho^{1 / 2}\right) \rightarrow 0, \rho \rightarrow 0 ; F(\rho) \rightarrow 0, \rho \rightarrow \infty .
$$

Note that the points $j, Z=0$ with any $j$ belongs to the nonsingular region, namely, to its lower boundary, which implies that the both total free Dirac Hamiltonians $\hat{H}_{s}^{1}, s= \pm 1$, are defined uniquely as s.a. operators in $\mathfrak{H}=L^{2}\left(\mathbb{R}^{2}\right) \oplus L^{2}\left(\mathbb{R}^{2}\right)$. Their spectra and (generalized) eigenfunctions are well known.

Turning to partial radial Hamiltonians $\hat{h}_{1}(Z, j, s)$ with $Z \neq 0$, we first point out that the nonsingularity condition $\gamma^{2}=j^{2}-g^{2} \geq 1 / 4$ excludes the values $j= \pm 1 / 2$ (we recall that the branches of the singular curve begin at the points $(j=1 / 2, Z=0)$ and $(j=-1 / 2, Z=0))$, and therefore, the partial radial Hamiltonians $\hat{h}_{\mathfrak{e}}(Z, \pm 1 / 2, s)$ with $Z \neq 0$ are not uniquely defined.

Before proceeding to spectra and (generalized) eigenfunctions of uniquely defined partial radial Hamiltonians $\hat{h}_{1}(Z, j, s)$ with $Z \neq 0$, and therefore with $|j|>1 / 2: j= \pm 3 / 2, \pm 5 / 2, \ldots$, we recall some notions related to a classification of the spectrum points of a s.a. operator following the conventional mathematical terminology, which does not unfortunately coincide with the physical one.

We call eigenvalues of a s.a. operator only those points of its spectrum which correspond to its bound states, the eigenstates whose wave functions (doublets) are square integrable. Note that in the physical literature, any 
spectrum point of a s.a. operator is often called its eigenvalue. Recall that in the physical literature, eigenvalues of Hamiltonians are conventionally called the energy levels.

The set of all eigenvalues of a given s.a. operator is called its point spectrum. The set of all isolated eigenvalues of a given s.a. operator is called its discrete spectrum, it is evidently a subset of the point spectrum unless they coincide. In the physical literature, the point and discrete spectrum are often identified.

The closure of the complement of the point spectrum in the whole spectrum of a s.a. operator is called its continuous spectrum. In the physical literature including textbooks, a rigorous definition of continuous spectrum is usually absent, it is replaced by heuristic arguments and examples.

It can happen that a spectrum point of a s.a. operator belongs to its point spectrum and to its continuous spectrum simultaneously. Such a spectrum point is not a point of the discrete spectrum.

The spectrum of each partial radial Hamiltonian $\hat{h}_{1}(Z, j, s)$ in the nonsingular region is simple (nondegenerate). It consists of a continuous spectrum occupying the both negative and positive semiaxis $(-\infty,-m]$ and $[m, \infty)$ and of a discrete spectrum located in the interval $(0, m)$ that consists of a growing infinite number of energy levels $E_{n}$, accumulated at the point $m$,

$$
\begin{aligned}
& \operatorname{spec} \hat{h}_{1}(Z, j, s)=\{E \in(-\infty,-m] \cup[m, \infty)\} \cup\left\{E_{n} \in(0, m)\right\}, \\
& E_{n}=E_{1 n}(Z, j, s)=\frac{(n+\gamma) m}{\sqrt{g^{2}+(n+\gamma)^{2}}}, \quad n \in \mathcal{N}_{\zeta}, \\
& \mathcal{N}_{\zeta}= \begin{cases}\mathbb{N}=\{1,2, \ldots\}, & \zeta=1, \quad \text { or } s j<0, \\
\mathbb{Z}_{+}=\{0,1,2, \ldots\}, & \zeta=-1, \quad \text { or } s j>0,\end{cases} \\
& \gamma=\sqrt{j^{2}-g^{2}} \geq 1 / 2, \quad j= \pm 3 / 2, \pm 5 / 2, \ldots
\end{aligned}
$$

The spectra of $\hat{h}_{1}(Z, j, s)$ can be obtained from the spectra of the corresponding radial Hamiltonians $\hat{h}_{1}(Z, j, \zeta)$ in the 3-dimensional case (constructed in Refs. [12, 13, 14, 16]) by the substitutions

$$
\begin{aligned}
& j=1 / 2,3 / 2, \cdots \rightarrow j= \pm 3 / 2, \pm 5 / 2, \ldots \\
& \zeta= \pm 1 \rightarrow \zeta=-s \operatorname{sgn}(j) \\
& \gamma=\sqrt{(j+1 / 2)^{2}-q^{2}} \rightarrow \gamma=\sqrt{j^{2}-g^{2}}
\end{aligned}
$$

We note that discrete energy levels with given $Z$ and $j$ are independent of $s$ and formally coincide for $s= \pm 1$, but the sets $\mathcal{N}_{\zeta}$, the sequences of integers $n$ labelling the energy levels, are different for different $s$, or equivalently, for different values of the variable $\zeta=-s \operatorname{sgn}(j)= \pm 1$, namely, the sequences differ by first terms; for brevity, we call these sets the sector $\zeta=+1$ and the sector $\zeta=-1$.

Normalized (generalized) eigenfunctions $U_{1 E}(\rho),|E| \geq m$, of continuous spectrum and normalized eigen- 
functions $U_{1 n}(\rho)$ of bound states of energy $E_{n}$ for the partial radial Hamiltonians $\hat{h}_{1}(Z, j, s)$ are given by

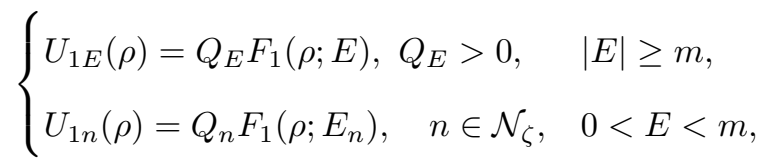

where the doublet $F_{1}(\rho ; E)$ is given by $(16)$ and 18$)$ and

$$
\begin{aligned}
& Q_{E}^{2}=\frac{2 \pi g^{2} k(|E|-\operatorname{sgn}(E) m)(2 k / m)^{2 \gamma} e^{\pi g E / k}}{\Gamma^{2}(2 \gamma+1)\left|\Gamma\left(-\gamma+i \frac{g}{k}|E|\right)\right|^{2}\left(\cosh \left(2 \pi \frac{g}{k} E\right)-\cos (2 \pi \gamma)\right)\left((\kappa+\gamma)^{2} k^{2}+g^{2}(E-m)^{2}\right)}, \\
& Q_{n}^{2}=\frac{\Gamma(2 \gamma+1+n) \tau_{n}^{3}\left(2 \tau_{n} / m\right)^{2 \gamma}}{m^{2} n ! \Gamma^{2}(2 \gamma+1)} \frac{g\left(m-E_{n}\right)-(\kappa-\gamma) \tau_{n}}{g\left(m-E_{n}\right)-(\kappa+\gamma) \tau_{n}}, \\
& k=\sqrt{E^{2}-m^{2}}, \quad \tau_{n}=g m\left[g^{2}+(n+\gamma)^{2}\right]^{-1 / 2},
\end{aligned}
$$

they form a complete orthonormalized system in $\mathbb{L}^{2}\left(\mathbb{R}_{+}\right)$in the sense of inversion formulas, see [13.

In conclusion, we point out that a remarkable equality $\hat{h}_{1}(Z, j, s)=\hat{h}_{1}(Z,-j,-s)$ takes place.

\subsection{Singular region}

In the singular region of the upper $(j, Z)$ plane, $Z>Z_{\mathrm{s}}(j)$, which is equivalent to $\Upsilon_{+}^{2}=j^{2}-g^{2}<1 / 4$, s.a. partial radial Hamiltonians $\hat{h}_{\mathfrak{e}}(Z, j, s)$ as s.a. extensions of the initial symmetric operators $\hat{h}_{\text {in }}(Z, j, s)$ are not defined uniquely for each triple $Z, j, s$. The reason is that the deficiency indices $m_{+}, m_{-}$of each symmetric operator $\hat{h}_{\text {in }}(Z, j, s)$ are $(1,1)$, and therefore, there exists a one-parameter family of its s.a. extensions, s.a. partial radial Hamiltonians, parametrized by the parameter $\nu \in[-\pi / 2, \pi / 2],-\pi / 2 \sim \pi / 2$, its own for each Hamiltonian. Partial radial Hamiltonians with the same triple $Z, j, s$, but with different $\nu$ are associated with the same differential operation $\check{h}(Z, j, s)$, but differ by their domains that are subdomains of the natural

domain $D_{\breve{h}(Z, j, s)}^{*}\left(\mathbb{R}_{+}\right)$for $\breve{h}(Z, j, s)$ specified by certain asymptotic s.a. boundary conditions at the origin which explicitly contain the parameter $\nu$.

As in the 3-dimensional Coulomb problem, we divide the singular region into the three subregions, the respective subcritical, critical, and overcritical regions, distinguished by a character of asymptotic s.a. boundary conditions at the origin.

We let $\hat{h}_{k \nu}, k=2,3,4$, denote s.a. partial radial Hamiltonians in the respective subcritical, $k=2$, critical, $k=3$, and overcritical, $k=4$, regions (for brevity, we here omit their arguments $Z, j, s$ of course, they are always implicitly implied). The s.a. boundary conditions specifying Hamiltonians $\hat{h}_{k \nu}$ are similar in each singular subregion, which provides a similar solution of the spectral problem for $\hat{h}_{k \nu}$ with a given $k$.

For completeness, we briefly remind the reader of the basic points of the spectral analysis of s.a. radial Hamiltonians $\hat{h}_{k \nu}$ based on the Krein method of guiding functionals, for details, see [13].

We say in advance that in all three singular subregions, there exists a unique guiding functional for each $\hat{h}_{k \nu}$, which implies that its spectrum is simple (nondegenerate in physical terminology). In such a case, the 
basic notion of spectral analysis is the spectral function $\sigma_{k \nu}(E), E \in \mathbb{R}$ is the energy variable, and especially its (generalized) derivative $\sigma_{k \nu}^{\prime}(E)$. By construction, the function $\sigma_{k \nu}^{\prime}(E)$ is given by

$$
\sigma_{k \nu}^{\prime}(E)=\frac{1}{\pi} \operatorname{Im} \frac{1}{\omega_{k \nu}(E+i 0)},
$$

where the function $\omega_{k \nu}(W), W=E+i y \in \mathbb{C}, y \neq 0$, is the certain function which comes from the Green function of the operator $\hat{h}_{k \nu}$, the kernel of the integral representation for the resolvent $\left(\hat{h}_{k \nu}-W\right)^{-1}$ of the operator $\hat{h}_{k \nu}$, namely, from a factor in the representation of the Green function in terms of products of a doublet $U_{k \nu}(\rho ; W)$ and the doublet $F_{3}(\rho ; W)$ 20). The doublet $U_{k \nu}(\rho ; W)$ is a linear combination of doublets $F_{1}(\rho ; W)$ and $F_{2}(\rho ; W)(18)$ that satisfies the asymptotic s.a. boundary conditions.

The spectrum of the operator $\hat{h}_{k \nu}$ is the support of the function $\sigma_{k \nu}^{\prime}(E)$, and the restriction $U_{k \nu}(\rho ; E)$ of the doublet $U_{k \nu}(\rho ; W)$ to the spectrum point $E$ of the operator $\hat{h}_{k \nu}$ is the corresponding eigenfunction of $\hat{h}_{k \nu}$.

In particular, a $\delta$ - function singularity of the function $\sigma_{k \nu}^{\prime}$ at some point $E_{n}$ caused by a simple zero of the real-valued function $\omega_{k \nu}(E)$ at this point, $\omega_{k \nu}\left(E_{n}\right)=0$ and $\operatorname{Im} \omega_{k \nu}(E)=0,\left|E-E_{n}\right|<\delta$, is a manifestation of the eigenvalue $E_{n}$ of the corresponding partial Hamiltonian.

The points $E$ where the function $\omega_{k \nu}(E)$ is nonzero, not real, and continuous are the points of the continuous spectrum of $\hat{h}_{k \nu}$. At such points, the function $\sigma_{k \nu}^{\prime}(E)$ is positive, $\sigma_{k \nu}^{\prime}(E)=Q_{k \nu}^{2}(E)>0$, and $Q_{k \nu}(E)=\sqrt{\sigma_{k \nu}^{\prime}(E)}$ is the normalization factor for the corresponding (generalized) eigenfunction $U_{k \nu}(\rho ; E)$ of continuous spectrum. We say in advance that in all three singular subregions, the continuous spectrum of each $\hat{h}_{k \nu}$ is the union $(-\infty,-m] \cup[m, \infty)$ of the two semiaxis.

In the interval $(-m, m)$, any function $\omega_{k \nu}(E)$ is real, but it has isolated simple zeroes at some points $E_{k n}(\nu)$, $\omega_{k \nu}\left(E_{k n}(\nu)\right)=0, n=1,2, \ldots$ (numbering can be different, see below). These points are the isolated eigenvalues of the operator $\hat{h}_{k \nu}$ forming its discrete spectrum, and the doublets $U_{k \nu}\left(\rho ; E_{k n}(\nu)\right)$ are the corresponding (normalizable) eigenfunctions. Really, in the vicinity of such points, the function $1 / \omega_{k \nu}(E+i 0)$ is of the form

$$
\frac{1}{\omega_{k \nu}(E+i 0)}=-\frac{Q_{k \nu, n}^{2}}{E-E_{k n}(\nu)+i 0}+O(1), \quad Q_{k \nu, n}^{2}=-\frac{1}{\omega_{k \nu}^{\prime}\left(E_{k n}(\nu)\right)}>0,
$$

so that $\sigma_{k \nu}^{\prime}(E)=Q_{k \nu, n}^{2} \delta\left(E-E_{k n}(\nu)\right)$, and $Q_{k \nu, n}>0$ is the normalization factor for the eigenfunction $U_{k \nu}\left(\rho ; E_{k n}(\nu)\right)$. We say in advance that in all three singular subregions, the discrete spectrum $\left\{E_{k n}(\nu)\right\}$ is a growing infinite set of eigenvalues, energy levels, accumulated at the point $E=m$.

What is remarkable is that for each family $\left\{\hat{h}_{k \nu}(Z, j, s)\right\}$ of partial radial Hamiltonians with a given $k$, there exists some value $\nu=\nu_{-m}$ of the extension parameter such that $\omega_{k \nu_{-m}}(-m)=0$, and the point $E=-m$ is an eigenvalue of the operator $\hat{h}_{k \nu_{-m}}$ with the corresponding normalizable eigenfunction $U_{k \nu_{-m}}(\rho ;-m)$, i.e., belongs to the point spectrum of $\hat{h}_{k \nu_{-m}}$. The peculiarity is that the point $E=-m$ also belongs to the continuous spectrum of $\hat{h}_{k \nu_{-m}}$ being the upper boundary of its lower branch. We thus encounter the case mentioned above where the Hamiltonian $\hat{h}_{k \nu_{-m}}$ has a nontrivial point spectrum which is not reduced to pure discrete one. 
The normalized (generalized) eigenfunctions $U_{k \nu, E}(\rho)=Q_{k \nu}(E) U_{k \nu}(\rho ; E)$ of continuous spectrum (in physical terminology, they "are normalized to $\delta$ function in energy") and normalized eigenfunctions $U_{k \nu, n}(\rho)=$ $Q_{k \nu, n} U_{k \nu}\left(\rho ; E_{k n}(\nu)\right)$ of discrete spectrum form a complete orthonormalized system in $\mathbb{L}^{2}\left(\mathbb{R}_{+}\right)$in the sense of inversion formulas. We call the function $\omega_{\nu k}(E)$ the basic function and the doublet $U_{k \nu}(\rho ; W)$ the basic doublet.

Note that the spectral analysis in the nonsingular region (it was omitted here, we present only its results) follow the same scheme with the basic function $\omega(W) / \Gamma(-2 \gamma)$, where the function $\omega(W)$ is given in $(20)$, instead of $\omega_{k \nu}(W)$ and basic doublet $F_{1}(\rho ; W)(18)$ instead of $U_{k \nu}(\rho ; W)$.

In what follows, we briefly outline the results of spectral analysis in each singular subregion including a specification of partial radial Hamiltonians $\hat{h}_{k \nu}$ and details of their point spectra.

\subsubsection{Subcritical region}

The subcritical region in the upper $(j, Z)$ half-plane is defined by the inequalities $Z_{\mathrm{s}}(j)<Z<Z_{\mathrm{c}}(j)$, which is equivalent to $0<\Upsilon_{+}=\gamma<1 / 2$, where $Z_{\mathrm{c}}(j)=\alpha_{F}^{-1} \epsilon|j|$, the latter is equivalent to $g=g_{\mathrm{c}}(j)=|j|$, or $\gamma=0$, see Fig. 1.

The value $Z_{\mathrm{c}}(j)$ can be called the critical $Z$-value for a given $j$. For illustration, we present the first $g_{\mathrm{c}}(j)$ values for first small half-integer $j$ :

$$
g_{\mathrm{c}}( \pm 1 / 2)=0.5 ; g_{\mathrm{c}}( \pm 3 / 2)=1.5 ; g_{\mathrm{c}}( \pm 5 / 2)=2.5
$$

In the subcritical region, the s.a. radial Hamiltonians $\hat{h}_{2 \nu}(Z, j, s)$ are specified by asymptotic s.a. boundary conditions at the origin of the form,

$$
\begin{aligned}
& F(\rho)=c\left[(m \rho)^{\gamma} d_{+} \cos \nu+(m \rho)^{-\gamma} d_{-} \sin \nu\right]+O\left(\rho^{1 / 2}\right), \\
& d_{ \pm}=(1,(\kappa \pm \gamma) / g)^{T}, \quad \rho \rightarrow 0,
\end{aligned}
$$

where $c$ is an arbitrary complex number. The domain $D_{h_{2 \nu}(Z, j, s)}$ of the Hamiltonian $\hat{h}_{2 \nu}(Z, j, s)$ is

$$
\left.D_{h_{2 \nu}(Z, j, s)}=\left\{F(\rho): \quad F(\rho) \in D_{h(Z, j, s)}^{*}\left(\mathbb{R}_{+}\right) \text {and } F \text { obey } 29\right\}\right\} .
$$

The basic function $\omega_{2 \nu}(W)$ and doublet $U_{2 \nu}(\rho ; W)$ are the respective

$$
\omega_{2 \nu}(W)=\frac{2 \gamma}{g} \frac{\omega(W) \cos \nu+g^{-1} \Gamma(1-2 \gamma) \sin \nu}{\omega(W) \sin \nu-g^{-1} \Gamma(1-2 \gamma) \cos \nu},
$$

where the function $\omega(W)$ is given in 20 , and

$$
U_{2 \nu}(\rho ; W)=F_{1}(\rho ; W) \cos \nu+F_{2}(\rho ; W) \sin \nu,
$$

where the doublets $F_{1}(\rho ; W)$ and $F_{2}(\rho ; W)$ are given by $(18)$. The derivative $\sigma_{2 \nu}^{\prime}(E)$ of spectral function is given by

$$
\sigma_{2 \nu}^{\prime}(E)=\frac{1}{\pi} \operatorname{Im} \frac{1}{\omega_{2 \nu}(E+i 0)} .
$$


It is easy to determine the support of $\sigma_{2 \nu}^{\prime}(E)$ and find that the simple spectrum of the Hamiltonian $\hat{h}_{2 \nu}(Z, j, s)$ is given by

$$
\operatorname{spec} \hat{h}_{2 \nu}(Z, j, s)=\{E \in(-\infty,-m] \cup[m, \infty)\} \cup\left\{E_{2 n}(\nu) \in[-m, m)\right\} .
$$

It consists of the continuous spectrum $(-\infty,-m] \cup[m, \infty)$ and the point spectrum. The point spectrum is a growing infinite sequence $\left\{E_{2 n}(\nu)=E_{2 n}(Z, j, s ; \nu)\right\}$ of the energy levels $E_{2 n}(Z, j, s ; \nu)$ that are the roots of the equation

$$
\omega_{2 \nu}(E)=\frac{2 \gamma}{g} \frac{\omega(E) \cos \nu+g^{-1} \Gamma(1-2 \gamma) \sin \nu}{\omega(E) \sin \nu-g^{-1} \Gamma(1-2 \gamma) \cos \nu}=0, \quad E \in[-m, m),
$$

located in the semiinterval $[-m, m)$ and accumulated at the point $E=m$. The infinite sequences $\{n\}$ of integers $n$ labelling the energy levels depend on $s$ (compare with the nonsingular region) and are defined more exactly below.

The normalized (generalized) eigenfunctions $U_{2 \nu, E}(\rho)$ of continuous spectrum and normalized eigenfunctions $U_{2 \nu, n}(\rho)$ of point spectrum given by

$$
\begin{aligned}
& U_{2 \nu, E}(\rho)=Q_{2 \nu}(E) U_{2 \nu}(\rho ; E)=Q_{2 \nu}(E)\left(F_{1}(\rho ; E) \cos \nu+F_{2}(\rho ; E) \sin \nu\right), \\
& E \in(-\infty,-m] \cup[m, \infty) ; \\
& U_{2 \nu, n}(\rho)=Q_{2 \nu, n} U_{2 \nu}\left(\rho ; E_{2 n}(\nu)\right)=Q_{2 \nu, n}\left(F_{1}\left(\rho ; E_{2 n}(\nu)\right) \cos \nu+F_{2}\left(\rho ; E_{2 n}(\nu)\right) \sin \nu\right),
\end{aligned}
$$

where

$$
Q_{2 \nu}(E)=\sqrt{\sigma_{2 \nu}^{\prime}(E)}, \quad Q_{2 \nu, n}=\sqrt{-\frac{1}{\omega_{2 \nu}^{\prime}\left(E_{2 n}(\nu)\right)}},
$$

form a complete orthonormalized system in $\mathbb{L}^{2}\left(\mathbb{R}_{+}\right)$in the sense of inversion formulas.

Explicit expressions for the spectrum and eigenfunctions, including an explicit solution of Eq. (30), can be obtained in the two cases: $\nu= \pm \pi / 2$ and $\nu=0$.

1) Let $\nu= \pm \pi / 2$. In this case, we have

$$
\left.\omega_{2 \nu}(W)\right|_{\nu= \pm \pi / 2}=\frac{2 \gamma}{g^{2}} \frac{\Gamma(1-2 \gamma)}{\omega(W)},\left.\quad U_{2 \nu}(\rho, W)\right|_{\nu= \pm \pi / 2}=F_{2}(\rho ; W) .
$$

As we already mentioned above, the basic function and doublet for the spectral analysis in the nonsingular region are $\omega(W) / \Gamma(-2 \gamma)$ and $F_{1}(\rho ; W)$. But

$$
\frac{2 \gamma}{g^{2}} \frac{\Gamma(1-2 \gamma)}{\omega(W)}=\left.\frac{\omega(W)}{\Gamma(-2 \gamma)}\right|_{\gamma \rightarrow-\gamma}
$$

as is easily verified, and $F_{2}(\rho ; W)=\left.F_{1}(\rho ; W)\right|_{\gamma \rightarrow-\gamma}$. It follows that all the results concerning the spectrum and eigenfunctions in our case can be obtained from the corresponding results for the nonsingular region, including (26), 27], by the formal substitution $\gamma \rightarrow-\gamma$. In particular, the discrete spectrum is given by

$$
\mathcal{E}_{2 n}=E_{2 n}( \pm \pi / 2)=\frac{(n-\gamma) m}{\sqrt{g^{2}+(n-\gamma)^{2}}}, \quad n \in \mathcal{N}_{\zeta},
$$


for $\mathcal{N}_{\zeta}$, see 26. A comment concerning an implicit dependence of discrete energy levels $\mathcal{E}_{2 n}$ on $s$, or equivalently on $\zeta$, via the sequences $\mathcal{N}_{\zeta}$ of integers $n$ labelling the energy levels is similar to that for formula 26.

2) Let $\nu=0$. In this case, we have

$$
\left.\omega_{2 \nu}(W)\right|_{\nu=0}=-\frac{2 \gamma}{\Gamma(1-2 \gamma)} \omega(W)=\frac{\omega(W)}{\Gamma(-2 \gamma)},\left.\quad U_{2 \nu}(\rho, W)\right|_{\nu=0}=F_{1}(\rho ; W) .
$$

It follows that in the case of $\nu=0$, all the results concerning the spectrum and eigenfunctions are the direct extension of the corresponding results, including (26), (27), 28) for the nonsingular region where $\gamma \geq 1 / 2$ to the subcritical region where $0<\gamma<1 / 2$.

A consideration of the general case $|\nu|<\pi / 2$ is completely similar to that for the singular region in the 3-dimensional Coulomb problem (see [13]). We cite only most important properties of the point spectrum. First, for each $Z, j, s$ and $\nu \neq \nu_{-m}$, see (33) just below, the point spectrum is a pure discrete one. At $\nu=\nu_{-m}$, the discrete spectrum is complemented by the energy level $E=-m$, which is simultaneously a point of continuous spectrum, the upper boundary of its lower branch. This $\nu_{-m}$ is determined from Eq. 30 by setting $E=-m$ and noting that $\omega(-m)=g^{-1} \Gamma(1+2 \gamma)(2 g)^{-2 \gamma}$ to yield

$$
\tan \nu_{-m}=-\frac{\Gamma(1+2 \gamma)}{\Gamma(1-2 \gamma)}(2 g)^{-2 \gamma} .
$$

It is remarkable that $\nu_{-m}=\nu_{-m}(Z, j)$ depends on $|j|$ and is independent of $s$.

For illustration, we give a graph (see Fig. 2) of the parameter $\nu_{-m}$ as function of $g$ for $j= \pm 1 / 2$.

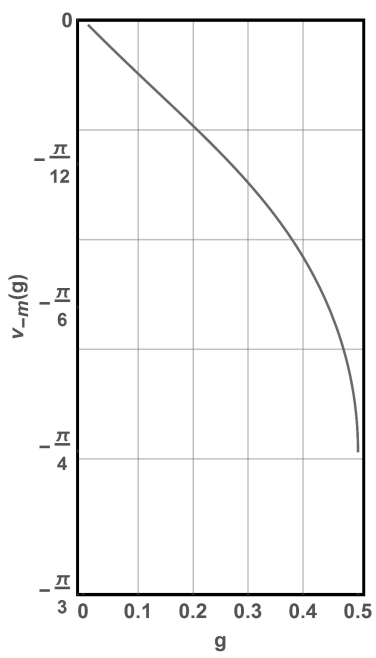

Figure 2: $g$-dependence of $\nu_{-m}$ for $j=1 / 2$.

Second, as follows from (9) and is confirmed by (26) and (32), we should distinguish the sectors $\zeta=1$ and $\zeta=-1$ of energy levels, in particular, due to the different sequences of integers $\mathcal{N}_{\zeta}$, see the comment to formula 267. What is more, the dependence of the energy levels $E_{2 n}(Z, j, s ; \nu)$ on $s$ in the general case of $0<|\nu|<\pi / 2$ becomes explicit. 
We let $n_{\zeta}$ denote the first term in the sequence $\mathcal{N}_{\zeta}: n_{1}=1, n_{-1}=0$. It should be emphasized that in each sector, there is the lowest energy level $-m$ corresponding to the same $\nu_{-m} \sqrt{33}$, in particular $\mathcal{E}_{2 n_{\zeta}}>-m$. We let $E_{2\left(n_{\zeta}-1\right)}\left(\nu_{-m}\right)$ denote this level.

In the energy semiinterval $\left[-m, \mathcal{E}_{2 n_{\zeta}}\right)$, there is one energy level $E_{2\left(n_{\zeta}-1\right)}(\nu)$ for each $\nu \in\left(-\pi / 2, \nu_{-m}\right]$ monotonically increasing from $-m$ to $\mathcal{E}_{2 n_{\zeta}}-0$ as $\nu$ goes from $\nu_{-m}$ to $-\pi / 2+0$, while for $\nu \in\left(\nu_{-m}, \pi / 2\right)$, there is no energy level. In each energy interval $\mathcal{E}_{2 n}, \mathcal{E}_{2(n+1)}, n \geq n_{\zeta}$, for each $\nu \in(-\pi / 2, \pi / 2)$ there is one energy level $E_{2 n}(\nu)$ monotonically increasing from $\mathcal{E}_{2 n}+0$ to $\mathcal{E}_{2(n+1)}-0$ as $\nu$ goes from $\pi / 2-0$ to $-\pi / 2+0$. It is worth noting that

$$
\lim _{\nu \rightarrow-\pi / 2} E_{2(n-1)}(\nu)=\lim _{\nu \rightarrow \pi / 2} E_{2 n}(\nu)=\mathcal{E}_{2 n}, \quad n \in \mathcal{N}_{\zeta} .
$$

For illustration, we give graphs of low energy levels $j=1 / 2$ with $g=0.4$ as functions of $\nu$ for $s=+1$ (Fig. 3a) and $s=-1$ (Fig. 3b).
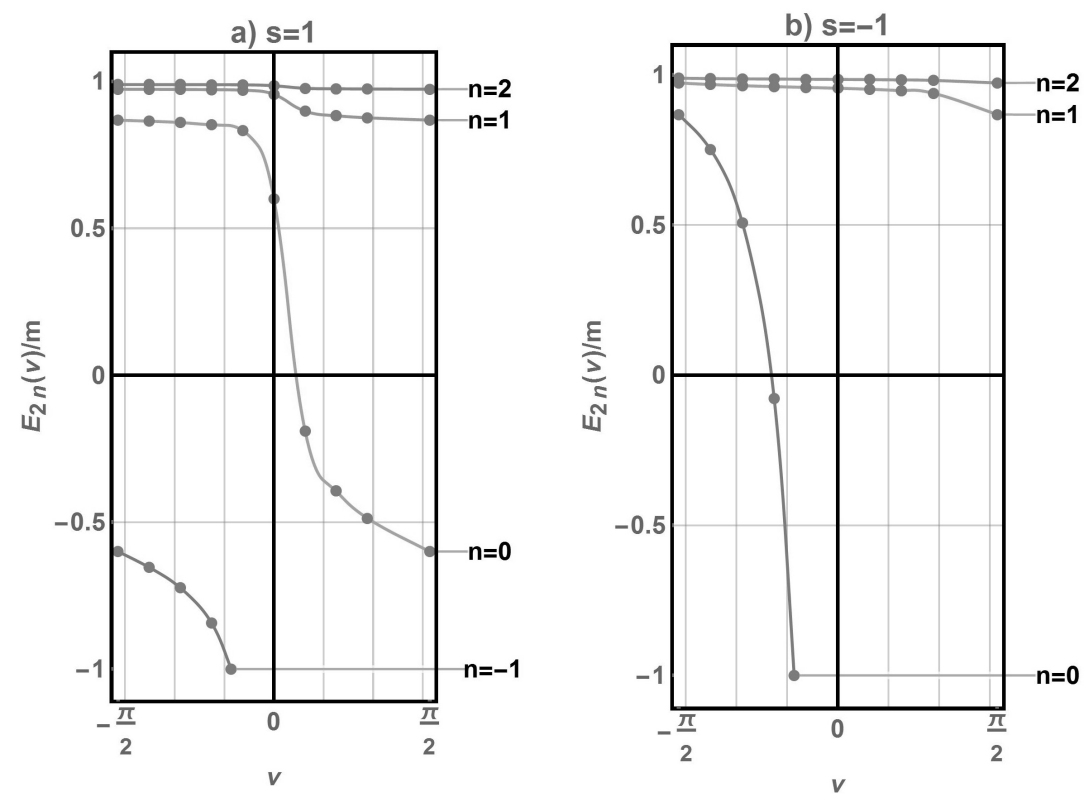

Figure 3: $\nu$-dependence of energy levels $E_{2 n}(\nu)$.

In conclusion, we point out that the remarkable equality

$$
\hat{h}_{2 \nu}(Z, j, s)=\hat{h}_{2 \nu}(Z,-j,-s)
$$

holds, in particular, because s.a. boundary conditions 29 are invariant under the simultaneous replacements $j \rightarrow-j, s \rightarrow-s$. 


\subsubsection{Critical region}

The critical region is the critical curve $Z=Z_{\mathrm{c}}(j)$, which is equivalent to $g=g_{\mathrm{c}}(j)$, or $\Upsilon_{+}=\gamma=0$. Note that this region is of academic interest in some sense: the physical values of the pairs $j$ (half-integer) and $Z$ (integer) lie on the critical curve for very special values of the graphene "fine structure constant " $\alpha_{F} / \epsilon, \alpha_{F} / \epsilon=|j| / Z$. In particular, if $\alpha_{F} / \epsilon$ is an irrational number, no physical pair $(j, Z)$ lies on the critical curve. In this region, the s.a. radial Hamiltonians $\hat{h}_{3 \nu}(Z, j, s)$ are specified by asymptotic s.a. boundary conditions at the origin of the form

$$
\begin{aligned}
& F(\rho)=c\left[d_{0}(\rho) \cos \nu+d_{+} \sin \nu\right]+O\left(\rho^{1 / 2} \log \rho\right), \rho \rightarrow 0, \\
& \nu \in[-\pi / 2, \pi / 2], \quad-\pi / 2 \sim \pi / 2,
\end{aligned}
$$

where the constant doublet $d_{+}=\left.d_{+}\right|_{\gamma=0}$ and the $\rho$ dependent doublet $d_{0}(\rho)$ are given in respective (21) and 23. The domain $D_{h_{3 \nu}(Z, j, s)}$ of the Hamiltonian $\hat{h}_{3 \nu}(Z, j, s)$ is

$$
\left.D_{h_{3 \nu}(Z, j, s)}=\left\{F(\rho): F(\rho) \in D_{h(Z, j, s)}^{*}\left(\mathbb{R}_{+}\right) \text {and } F \text { obey } 34\right\}\right\} .
$$

The basic function $\omega_{3 \nu}(W)$ is

$$
\omega_{3 \nu}(W)=\frac{f(W) \cos \nu-\sin \nu}{g_{\mathrm{c}}(j)[f(W) \sin \nu+\cos \nu]},
$$

where the function $f(W)$ is given by $(25)$, and the basic doublet $U_{3 \nu}(W)$ is

$$
U_{3 \nu}(W)=F_{1}^{(0)}(\rho, W) \sin \nu+F_{2}^{(0)}(\rho, W) \cos \nu,
$$

where the doublets $F_{1}^{(0)}(\rho, W)$ and $F_{2}^{(0)}(\rho, W)$ are given by the respective 21) and 22, 23, while the role of the doublet $F_{3}(\rho, W)$ plays the doublet $F_{3}^{(0)}(\rho, W)$ given by 24,25 . The derivative $\sigma_{3 \nu}^{\prime}(E)$ of the spectral function is given by

$$
\sigma_{3 \nu}^{\prime}(E)=\frac{1}{\pi} \operatorname{Im} \frac{1}{\omega_{3 \nu}(E+i 0)} .
$$

A knowledge of the function $\sigma_{3 \nu}^{\prime}(E)$ requires a knowledge of the reduction $f(E)=f(E+i 0)$ of the function $f(W)$ 24 to the real axis. For $|E| \geq m$, it is given by

$$
f(E)=\ln \left(2 e^{-i \epsilon \pi / 2} \frac{k}{m}\right)+\psi\left(-i g_{\mathrm{c}}(j) \frac{|E|}{k}\right)+\frac{\zeta(E-m)+i \epsilon k}{2 g_{\mathrm{c}}(j) E}-2 \psi(1),|E| \geq m,
$$

while for $|E|<m$, it is given by

$$
f(E)=\ln \left(2 \frac{\tau}{m}\right)+\psi\left(-g_{\mathrm{c}}(j) \frac{E}{\tau}\right)+\frac{\zeta(E-m)-\tau}{2 g_{\mathrm{c}}(j) E}-2 \psi(1), \quad|E|<m,
$$

where

$$
k=\sqrt{E^{2}-m^{2}}, \quad \tau=\sqrt{m^{2}-E^{2}}, \quad \epsilon=\operatorname{sgn}(E), \quad \zeta=\zeta(j, s), \quad \psi(z)=\Gamma^{\prime}(z) / \Gamma(z) .
$$


Determining the support of $\sigma_{3 \nu}^{\prime}(E)$ and evaluating the relevant quantities (which is rather tedious) results in the conclusion that the simple spectrum of the Hamiltonian $\hat{h}_{3 \nu}(Z, j, s)$ is given by

$$
\operatorname{spec} \hat{h}_{3 \nu}(Z, j, s)=\{E \in(-\infty,-m] \cup[m, \infty)\} \cup\left\{E_{3 n}(\nu) \in[-m, m)\right\},
$$

it consists of the continuous spectrum $(-\infty,-m] \cup[m, \infty)$ and the point spectrum. The point spectrum is a growing infinite sequence $\left\{E_{3 n}(\nu)=E_{3 n}(Z, j, s ; \nu)\right\}$ of the energy levels $E_{3 n}(Z, j, s ; \nu)$ that are the roots of the equation $\omega_{3 \nu}(E)=0, E \in[-m, m)$, which is equivalent to

$$
\frac{f(E) \cos \nu-\sin \nu}{f(E) \sin \nu+\cos \nu}=0, \quad|E|<m,
$$

they are located in the semiinterval $[-m, m)$ and are accumulated at the point $E=m$. The infinite sequences $\{n\}$ of integers $n$ labelling the discrete energy levels depend on $s$ and are defined more exactly below.

It is also evident from (37) and (36) that energy levels with given $(Z, j)$ explicitly depend on $\zeta$, i.e., on $s$. The normalized (generalized) eigenfunctions $U_{3 \nu, E}(\rho)$ of continuous spectrum and normalized eigenfunctions $U_{3 \nu, n}(\rho)$ of point spectrum given by

$$
\begin{aligned}
& U_{3 \nu, E}(\rho)=Q_{3 \nu}(E) U_{3 \nu}(\rho ; E)=Q_{3 \nu}(E)\left(F_{1}^{(0)}(\rho ; E) \cos \nu+F_{2}^{(0)}(\rho ; E) \sin \nu\right), \\
& E \in(-\infty,-m] \cup[m, \infty) ; \\
& U_{3 \nu, n}(\rho)=Q_{3 \nu, n} U_{3 \nu}\left(\rho ; E_{3 n}(\nu)\right)=Q_{3 \nu, n}\left(F_{1}^{(0)}\left(\rho ; E_{2 n}(\nu)\right) \cos \nu+F_{2}^{(0)}\left(\rho ; E_{2 n}(\nu)\right) \sin \nu\right),
\end{aligned}
$$

where

$$
Q_{3 \nu}(E)=\sqrt{\sigma_{3 \nu}^{\prime}(E)}, \quad Q_{3 \nu, n}=\sqrt{-\frac{1}{\omega_{3 \nu}^{\prime}\left(E_{3 n}(\nu)\right)}},
$$

form a complete orthonormalized system in $\mathbb{L}^{2}\left(\mathbb{R}_{+}\right)$in the sense of inversion formulas.

In the case of $\nu= \pm \pi / 2$, we obtain explicit formulas for the spectrum and eigenfunctions. In this case, we have

$$
\begin{aligned}
& \left.\omega_{3 \nu}(W)\right|_{\nu= \pm \pi / 2}=-\frac{1}{g_{\mathrm{c}}(j) f(W)}, \\
& \left.U_{3 \nu}(W)\right|_{\nu= \pm \pi / 2}=F_{1}^{(0)}(\rho, W)=\left.F_{1}(\rho, W)\right|_{\gamma=0}, \\
& \left.\sigma_{3 \nu}^{\prime}(E)\right|_{\nu= \pm \pi / 2}=-\frac{g_{\mathrm{c}}(j)}{\pi} \operatorname{Im} f(E+i 0) .
\end{aligned}
$$

In the range $|E| \geq m$ of the continuous spectrum, taking $f(E)$ (35) and using the relation $\psi(z)-\psi(-z)=$ $-\pi \cot (\pi z)-z^{-1}$, we have

$$
\left.\sigma_{3 \nu}^{\prime}(E)\right|_{\nu= \pm \pi / 2}=\left.Q_{3 \nu}^{2}(E)\right|_{\nu= \pm \pi / 2}=\frac{g_{\mathrm{c}}(j)}{2}\left(\operatorname{coth}\left(\pi g_{\mathrm{c}}(j) \frac{|E|}{k}\right)+\epsilon\right), \quad|E| \geq m
$$

Note that $\left.\sigma_{3 \nu}^{\prime}(E)\right|_{\nu= \pm \pi / 2} \geq 0$ for $|E| \geq m$, as it must, and the normalization factor $\left.Q_{3 \nu}^{2}(E)\right|_{\nu= \pm \pi / 2}$ for the eigenfunctions $\left.U_{3 \nu}(\rho ; E)\right|_{\nu= \pm \pi / 2}=F_{1}^{(0)}(\rho, E)$ of continuous spectrum is nonnegative. 
In the range $|E|<m$, taking $f(E)(36)$ and having regard to that the poles of $\left.\sigma_{3 \nu}^{\prime}(E)\right|_{\nu= \pm \pi / 2}$ determining the energy levels of the Hamiltonians are provided by the poles of $\psi(-z)$ at the points $z_{n}=n, n \in \mathbb{Z}_{+}$, and the pole of the third term with $\zeta=1$ in the r.h.s. of $(36)$ at the point $E=0$, we obtain that

$$
\left.\sigma_{3 \nu}^{\prime}(E)\right|_{\nu= \pm \pi / 2}=\left.\sum_{n \in \mathcal{N}_{\zeta}} Q_{3 \nu, n}^{2}\right|_{\nu= \pm \pi / 2} \delta\left(E-\mathcal{E}_{3 n}\right),
$$

where the normalization factors $\left.Q_{3 \nu, n}\right|_{\nu= \pm \pi / 2}$ for the eigenfunctions $\left.U_{3 \nu}\left(\rho ; \mathcal{E}_{3 n}\right)\right)\left.\right|_{\nu= \pm \pi / 2}=F_{1}^{(0)}\left(\rho, \mathcal{E}_{3 n}\right)$ of bound states are

$$
\left.Q_{3 \nu, n}\right|_{\nu= \pm \pi / 2}=\frac{\tau_{n}^{3 / 2}}{m}, \tau_{n}=\frac{g_{\mathrm{c}}(j) m}{\sqrt{g_{\mathrm{c}}^{2}(j)+n^{2}}}, n \in \mathcal{N}_{\zeta},
$$

and the corresponding discrete energy levels $\mathcal{E}_{3 n}=E_{3 n}(\nu= \pm \pi / 2)$ are

$$
\mathcal{E}_{3 n}=\frac{n m}{\sqrt{g_{\mathrm{c}}^{2}(j)+n^{2}}}, n \in \mathcal{N}_{\zeta} .
$$

Note that all the results for the spectrum and eigenfunctions in the critical region, $\gamma=0$, in the case of $\nu= \pm \pi / 2$ are obtained from the corresponding results in the nonsingular region, $\nu>1 / 2$, including (26), 227), 28), by formally continuing the latter to the point $\gamma=0$.

As to the general case $|\nu|<\pi / 2$, we cite only most important properties of the discrete spectrum. An exposition is much like that in the subcritical region.

First, for each $j, s$ in the critical region (where $Z$ is uniquely determined by $j$ ), and $\nu \neq \nu_{-m}$, see (38) just below, the point spectrum is a pure discrete one. At $\nu=\nu_{-m}$, the discrete spectrum is complemented by the energy level $E=-m$, which is simultaneously a point of continuous spectrum, the upper boundary of its lower branch. This $\nu_{-m}=\nu_{-m}(|j|, \zeta)$ is determined from Eq. (37) by setting $E=-m$ and noting that $f(-m)=\ln \left(2 g_{\mathrm{c}}(j)\right)-2 \psi(1)+\zeta / g_{\mathrm{c}}(j)$ to yield

$$
\tan \nu_{-m}(|j|, \zeta)=\ln \left(2 g_{\mathrm{c}}(j)\right)-2 \psi(1)+\frac{\zeta}{g_{\mathrm{c}}(j)} .
$$

It is remarkable that $\nu_{-m}$ as a function of $j$ and $\zeta$ depends on $|j|$, while as a function of $j$ and $s$, it depends on the both $j$ and $s$. It is also worth noting that $\nu_{-m}(|j|, \zeta)$ satisfies the inequalities

$$
\begin{aligned}
& \nu_{-m}(|j|, 1)>\nu_{-m}(|j|,-1), \quad \nu_{-m}(|j|, 1)>0, \quad \forall j, \\
& \nu_{-m}(1 / 2,-1)<0 ; \nu_{-m}(|j|,-1)>0, \quad|j|>3 / 2 .
\end{aligned}
$$

Second, at given $j, \zeta$, in the energy semiinterval $\left[-m, \mathcal{E}_{2 n_{\zeta}}\right.$ ) (we recall that $n_{\zeta}$ is equal to 1 for $\zeta=1$ and to 0 for $\zeta=-1)$, for each $\nu \in\left(-\pi / 2, \nu_{-m}(|j|, \zeta)\right], \nu_{-m}(|j|, \zeta)$ is given by (38), there is one energy level $E_{3\left(n_{\zeta}-1\right)}(\nu)$ monotonically increasing from $-m$ to $\mathcal{E}_{3 n_{\zeta}}-0$ as $\nu$ goes from $\nu_{-m}(|j|, \zeta)$ to $-\pi / 2+0$, while for $\nu \in\left(\nu_{-m}(|j|, \zeta), \pi / 2\right)$, there is no energy level. In each energy interval $\left(\mathcal{E}_{3 n}, \mathcal{E}_{3(n+1)}\right), n \geq n_{\zeta}$, for each $\nu \in(-\pi / 2, \pi / 2)$, there is one energy level $E_{3 n}(\nu)$ monotonically increasing from $\mathcal{E}_{3 n}+0$ to $\mathcal{E}_{3(n+1)}-0$ as $\nu$ goes from $\pi / 2-0$ to $-\pi / 2+0$. Note that relations

$$
\lim _{\nu \rightarrow-\pi / 2} E_{3(n-1)}(\nu)=\lim _{\nu \rightarrow \pi / 2} E_{3 n}(\nu)=\mathcal{E}_{3 n}, \quad n \in \mathcal{N}_{\zeta}
$$


hold.

For illustration, we give graphs of low energy levels $j=1 / 2$ with $g=0.5$ as functions of $\nu$ for $s=+1$ (Fig. 4a) and for $s=-1$ (Fig. 4b).
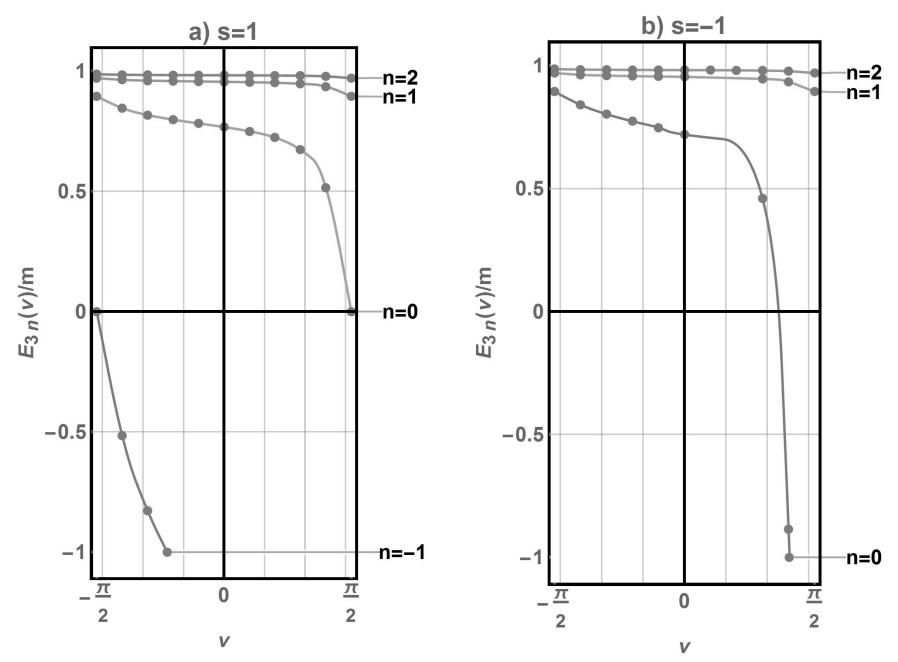

Figure 4: $\nu$-dependence of energy levels $E_{3 n}(\nu)$.

In conclusion, we point out that the remarkable equality $\hat{h}_{3 \nu}(Z, j, s)=\hat{h}_{3 \nu}(Z,-j,-s)$ holds, in particular, because s.a. boundary conditions (34) are invariant under the simultaneous replacements $j \rightarrow-j, s \rightarrow-s$.

\subsubsection{Overcritical region}

The overcritical region in the upper $(j, Z)$ half-plane is defined by the inequality $Z>Z_{c}(j)=\alpha^{-1} \epsilon|j|$, which is equivalent to $\Upsilon_{+}=i \sigma, \sigma=\sqrt{g^{2}-j^{2}}>0$. In this region, the s.a. radial Hamiltonians $\hat{h}_{4 \nu}(Z, j, s)$ are specified by the asymptotic s.a. boundary conditions at the origin of the form

$$
\begin{aligned}
& F(\rho)=c\left(i e^{i \nu}(m \rho)^{i \sigma} d_{+}-i e^{-i \nu}(m \rho)^{-i \sigma} d_{-}\right)+O\left(\rho^{1 / 2}\right), \quad d_{ \pm}=(1,(\kappa \pm i \sigma) / g)^{T}, \quad \rho \rightarrow 0, \\
& \nu \in[-\pi / 2, \pi / 2], \quad-\pi / 2 \sim \pi / 2 .
\end{aligned}
$$

The domain $D_{h_{4 \nu}(Z, j, s)}$ of the Hamiltonian $\hat{h}_{4 \nu}(Z, j, s)$ is

$$
D_{h_{4 \nu}(Z, j, s)}=\left\{F(\rho): \quad F(\rho) \in D_{\breve{h}(Z, j, s)}^{*}\left(\mathbb{R}_{+}\right) \text {and } F \text { obey } 39\right\} .
$$

The basic function $\omega_{4 \nu}(W)$ and doublet $U_{4 \nu}(W)$ are given by the respective

$$
\omega_{4 \nu}(W)=-\frac{4 i \sigma}{g} \frac{1-\frac{g}{\Gamma(1-2 i \sigma)} \omega(W) e^{2 i \nu}}{1+\frac{g}{\Gamma(1-2 i \sigma)} \omega(W) e^{2 i \nu}},
$$

where the function $\omega(W)$ is given in 20$)$, and $U_{4 \nu}(W)=i e^{i \nu} F_{1}(\rho ; W)-i e^{-i \nu} F_{2}(\rho ; W)$, where the doublets $F_{1}(\rho ; W), F_{2}(\rho ; W)$ are given by $(18)$. The derivative $\sigma_{4 \nu}^{\prime}(E)$ of the spectral function is given by

$$
\sigma_{4 \nu}^{\prime}(E)=\frac{1}{\pi} \operatorname{Im} \frac{1}{\omega_{4 \nu}(E+i 0)} \text {. }
$$


Determining the support of $\sigma_{4 \nu}^{\prime}(E)$ and evaluating the relevant quantities results in the conclusion that the simple spectrum of the Hamiltonian $\hat{h}_{4 \nu}(Z, j, s)$ is given by

$$
\operatorname{spec} \hat{h}_{4 \nu}(Z, j, s)=\{E:|E| \geq m\} \cup\left\{E_{4 n}(\nu) \in[-m, m)\right\}
$$

it consists of the continuous spectrum $(-\infty,-m] \cup[m, \infty)$ and the point spectrum. The point spectrum is a growing infinite sequence $\left\{E_{4 n}(\nu)=E_{4 n}(Z, j, s ; \nu)\right\}$ of the energy levels $E_{4 n}(Z, j, s ; \nu)$ that are the roots of the equation

$$
\omega_{4 \nu}(E)=-\frac{4 i \sigma}{g} \frac{1-\frac{g}{\Gamma(1-2 i \sigma)} \omega(E) e^{2 i \nu}}{1+\frac{g}{\Gamma(1-2 i \sigma)} \omega(E) e^{2 i \nu}}=0, \quad E \in[-m, m),
$$

which is equivalent to the equation, we call it the spectral equation for brevity,

$$
1-\frac{g \omega(E) e^{2 i \nu}}{\Gamma(1-2 i \sigma)}=0, \quad E \in[-m, m) .
$$

These energy levels are located in the semiinterval $[-m, m)$ and are accumulated at the point $E=m$. The infinite sequences $\{n\}$ of integers $n$ labelling the discrete energy levels are defined more exactly below. It is evident that energy levels with given $(Z, j)$ explicitly depend on $s$, or $\zeta$, in view of an explicit dependence of the function $\omega(E)$ on $s$.

The spectral equation (40) allows another form more convenient for a further analysis. It is sufficient to note that

$$
\begin{gathered}
-\frac{g \omega(E)}{\Gamma(1-2 i \sigma)}=\frac{\Gamma(2 i \sigma) \Gamma\left(-i \sigma-g \frac{E}{\tau}\right)[\tau(\kappa+i \sigma)-g(m-E)]}{\Gamma(-2 i \sigma) \Gamma\left(i \sigma-g \frac{E}{\tau}\right)[\tau(\kappa-i \sigma)-g(m-E)]}\left(\frac{2 \tau}{m}\right)^{-2 i \sigma}=e^{-2 i \Theta(E)}, \\
\Theta(E)=\sigma \ln \frac{2 \tau}{m}+\frac{1}{2 i}\left\{\ln \Gamma(-2 i \sigma)-\ln \Gamma(2 i \sigma)+\ln \Gamma\left(i \sigma-g \frac{E}{\tau}\right)-\ln \Gamma\left(-i \sigma-g \frac{E}{\tau}\right)\right. \\
\left.+\ln \left[\tau\left(g_{c}-i \zeta \sigma\right)-\zeta g(m-E)\right]-\ln \left[\tau\left(g_{c}+i \zeta \sigma\right)-\zeta g(m-E)\right]\right\},
\end{gathered}
$$

and Eq. 40 becomes $\cos [\Theta(E)-\nu]=0$. Note that $\Theta(E)$ is a smooth function on $(-m, m)$.

The normalized (generalized) eigenfunctions $U_{4 \nu, E}(\rho)$ of continuous spectrum and normalized eigenfunctions $U_{4 \nu, n}(\rho)$ of discrete spectrum given by

$$
\begin{aligned}
& U_{4 \nu, E}(\rho)=Q_{4 \nu}(E) U_{4 \nu}(\rho ; E)=Q_{4 \nu}(E)\left(i e^{i \nu} F_{1}(\rho ; E)-i e^{-i \nu} F_{2}(\rho ; E)\right), \\
& E \in(-\infty,-m] \cup[m, \infty) ; \\
& U_{4 \nu, n}(\rho)=Q_{4 \nu, n} U_{4 \nu}\left(\rho ; E_{4 n}(\nu)\right)=Q_{4 \nu, n}\left(i e^{i \nu} F_{1}\left(\rho ; E_{4 n}(\nu)\right)-i e^{-i \nu} F_{2}\left(\rho ; E_{4 n}(\nu)\right)\right),
\end{aligned}
$$

where

$$
Q_{4 \nu}(E)=\sqrt{\sigma_{4 \nu}^{\prime}(E)}, \quad Q_{4 \nu, n}=\sqrt{-\frac{1}{\omega_{4 \nu}^{\prime}\left(E_{4 n}(\nu)\right)}},
$$

form a complete orthonormalized system in $\mathbb{L}^{2}\left(\mathbb{R}_{+}\right)$in the sense of inversion formulas. 
We now refine a description of the discrete spectrum.

First, for each $Z, j, s$ in the overcritical region and $\nu \neq \nu_{-m}$, see 42 just below, the point spectrum is a pure discrete one. At $\nu=\nu_{-m}$, the discrete spectrum is complemented by the energy level $E=-m$, which is simultaneously a point of continuous spectrum, the upper boundary of its lower branch. This $\nu_{-m}(Z, j)$ is determined from Eq. 40 by setting $E=-m$ and noting that $g \omega(-m)=\Gamma(1+2 i \sigma)(2 g)^{-2 i \sigma}$ to yield

$$
e^{2 i \nu_{-m}}=e^{-i \pi}(2 g)^{2 i \sigma} \frac{\Gamma(-2 i \sigma)}{\Gamma(2 i \sigma)}, \quad \nu_{-m} \in[-\pi / 2, \pi / 2], \quad-\pi / 2 \sim \pi / 2 .
$$

It is remarkable that $\nu_{-m}(Z, j)$ does not depend on $s$.

For illustration, we give a graph (Fig. 5) of the parameter $\nu_{-m}$ as function of $g$ for $j=1 / 2$.

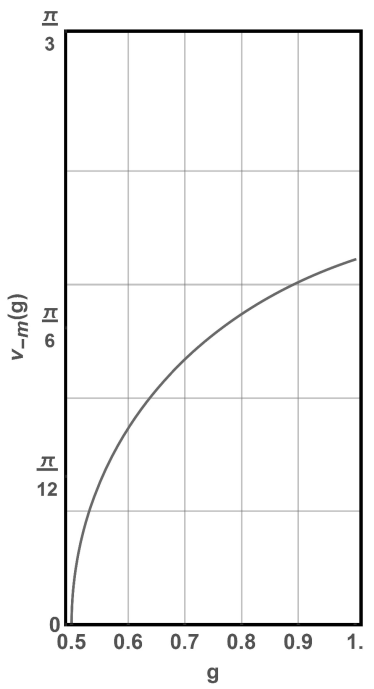

Figure 5: $g$-dependence of $\nu_{-m}$ for $j=1 / 2$.

Second, in contrast to the previous regions, a labelling of the energy levels in the overcritical region is independent of $\zeta$, i.e., it is the same for $\zeta=1$ and $\zeta=-1$.

Third, to describe the point spectrum in this region, it appears convenient to introduce a specific double labelling of the discrete energy levels with $\nu= \pm \pi / 2$. We formally distinguish values $\nu=-\pi / 2$ and $\nu=\pi / 2$ labelling the energy levels with $\nu=-\pi / 2$ by nonnegative integers, $E_{4 n}(-\pi / 2), n \in \mathbb{Z}_{+}=\{0,1,2,3, \ldots\}$, while the energy levels with $\nu=\pi / 2$ are labelled by positive integers, $E_{4 n}(\pi / 2), n \in \mathbb{N}_{+}=\{1,2,3, \ldots\}$. This artificial difference is actually erased under the identification $E_{4 n}(-\pi / 2)=E_{4(n+1)}(\pi / 2), n \in \mathbb{Z}_{+}$.

After this agreement, the point spectrum of Hamiltonians $\hat{h}_{4 \nu}(Z, j, s)$ looks as follows. In the energy interval $\left[-m, E_{40}(-\pi / 2)=E_{41}(\pi / 2)\right)$, there are no energy levels for $\nu \in\left(\nu_{-m}, \pi / 2\right)$, while for any $\nu \in\left(-\pi / 2, \nu_{-m}\right]$, there is one energy level $E_{40}(\nu)$ which increases monotonically from $-m$ to $E_{40}(-\pi / 2)-0$ as $\nu$ goes from $\nu_{-m}$ to $-\pi / 2+0$. In each energy interval $\left[E_{4 n}(\pi / 2), E_{4(n+1)}(\pi / 2)=E_{4 n}(-\pi / 2)\right), n \in \mathbb{N}_{+}$, there is one energy level $E_{4 n}(\nu)$, which increases monotonically from $E_{4 n}(\pi / 2)$ to $E_{4 n}(-\pi / 2)-0$ as $\nu$ goes from $\pi / 2$ to $-\pi / 2+0$. In 
particular, we have $-m \leq E_{40}(-\pi / 2)<E_{4 n}(-\pi / 2)<E_{4(n+1)}(-\pi / 2)<m, \forall n \in \mathbb{Z}_{+}$.

For illustration, we give graphs of low energy levels $j=1 / 2$ with $g=0.7$ as functions of $\nu$ for $s=+1$ (Fig. 6 a) and for $s=-1$ (Fig. 6 b).
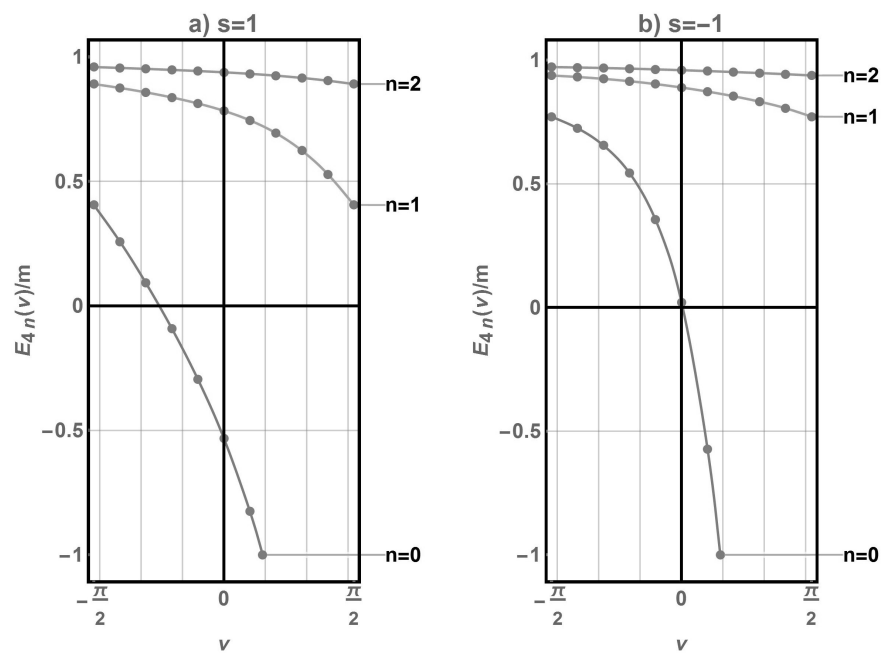

Figure 6: $\nu$-dependence of energy levels $E_{4 n}(\nu)$.

In conclusion, we point out that the remarkable equality

$$
\hat{h}_{4 \nu}(Z, j, s)=\hat{h}_{4 \nu}(Z,-j,-s)
$$

holds, in particular, because s.a. boundary conditions (39) are invariant under the simultaneous replacements $j \rightarrow-j, s \rightarrow-s$

\section{Self-adjoint total Hamiltonians and their spectra for point-like case}

In sect. 5.1 and 5.2 we constructed all s.a. partial radial Hamiltonians $\hat{h}_{\mathfrak{e}}(Z, j, s)$ for all values of $Z$ as s.a. extensions of the initial symmetric operators $\hat{h}_{i n}(Z, j, s)$ for any $j, s$ and solved spectral problems for all these Hamiltonians. As a result, 10 allow one to restore all s.a. operators $\hat{H}_{s}^{\mathfrak{e}}$ associated with the differential operation (4) for any $g$ and to describe the solution of the corresponding spectral problems for all the Hamiltonians $\hat{H}_{s}^{\mathfrak{e}}$.

It is convenient to introduce charge ranges in which the spectral problem has a similar description. These ranges are defined by characteristic points $k=l+1 / 2, l \in \mathbb{Z}_{+}$. The functions $g_{\mathrm{c}}(k)$ and $g_{\mathrm{s}}(k)$ are defined on this set by

$$
g_{\mathrm{c}}(k)=k, \quad g_{\mathrm{s}}(k+1)=\sqrt{(k+1)^{2}-\frac{1}{4}},
$$


and satisfy the following inequalities:

$$
g_{\mathrm{c}}(k)<g_{\mathrm{s}}(k+1)<g_{\mathrm{c}}(k+1)<g_{\mathrm{s}}(k+2) .
$$

Let us introduce intervals $\Delta(k)$ as follows:

$$
\begin{aligned}
& \Delta(0)=\left(0 ; \frac{1}{2}\right), \quad \Delta(k)=\left(g_{\mathrm{c}}(k), g_{\mathrm{c}}(k+1)\right]=(k, k+1], \\
& (0, \infty)=\Delta(0) \cup\left\{g_{\mathrm{c}}\left( \pm \frac{1}{2}\right)\right\} \cup\left(\bigcup_{k} \Delta(k)\right) .
\end{aligned}
$$

In turn, due to 43 , each interval $\Delta(k)$ can be represented as $\Delta(k)=\cup_{i=1,2,3} \Delta_{i}(k)$, where

$$
\Delta_{1}(k)=\left(g_{\mathrm{c}}(k), g_{\mathrm{s}}(k+1)\right], \quad \Delta_{2}(k)=\left(g_{\mathrm{s}}(k+1), g_{\mathrm{c}}(k+1)\right), \quad \Delta_{3}(k)=\left\{g_{\mathrm{c}}(k+1)\right\} .
$$

According to this division, we define three ranges $G_{i}=\bigcup_{k} \Delta_{i}(k), i=1,2,3$, of coupling parameters $g$, such that any given $g>g_{\mathrm{c}}( \pm 1 / 2)=1 / 2$ generates a pair of two integers, $k$ and $i=1,2,3$, such that $g \Longrightarrow(k, i)$, $g \in G_{i}$. Then, as follows from the consideration represented in Sect. 5.1-5.2, we obtain the following picture.

A. Let $g \Longrightarrow(k, 1)$, that is, $g \in \Delta_{1}(k)$ for some $k$, which means that

$$
k=g_{\mathrm{c}}(k)<g \leq g_{\mathrm{s}}(k+1)=\sqrt{(k+1)^{2}-\frac{1}{4}} .
$$

Consider quantum numbers $|j| \leq k$. Then $g>g_{\mathrm{c}}(k) \geq g_{\mathrm{c}}(j)$, which means that $g>g_{\mathrm{c}}(j)$. Such quantum numbers $j$ are characteristic for the overcritical region considered in Sect 5.2 .3 .

Consider quantum numbers $|j| \geq k+1$. Then $g \leq g_{\mathrm{s}}(k+1) \leq g_{\mathrm{s}}(j)$. Such quantum numbers $j$ are characteristic for the nonsingular region considered in Sect 5.1 .

Therefore, for such coupling $g$, we have

$$
\begin{aligned}
& U_{E}(\rho)=\left\{\begin{array}{ll}
U_{4 \nu, E}(\rho), & |j| \leq k, \\
U_{1 E}(\rho), & |j| \geq k+1,
\end{array} \quad|E| \geq m,\right. \\
& U_{n}(\rho)=\left\{\begin{array}{ll}
U_{4 \nu, n}(\rho), & |j| \leq k, \\
U_{1 n}(\rho), & |j| \geq k+1,
\end{array} \quad E_{n}= \begin{cases}E_{4 n}(\nu), & |j| \leq k, \\
E_{1 n}(Z, j, s), & |j| \geq k+1,\end{cases} \right.
\end{aligned}
$$

B. Let $g \Longrightarrow(k, 2)$, that is, $g \in \Delta_{2}(k)$ for some $k$, which means that

$$
\sqrt{(k+1)^{2}-\frac{1}{4}}=g_{\mathrm{s}}(k+1)<g<g_{\mathrm{c}}(k+1)=k+1 .
$$

Consider quantum numbers $|j| \leq k$. Then $g>g_{\mathrm{s}}(k+1)>g_{\mathrm{c}}(k) \geq g_{\mathrm{c}}(j)$, which means that $g>g_{\mathrm{c}}(j)$. Such quantum numbers $j$ are characteristic for the overcritical region considered in Sect 5.2 .3 .

Consider quantum numbers $|j|=k+1$. In this case $g_{\mathrm{s}}(j)<g<g_{\mathrm{c}}(j)$. Such quantum numbers $j$ are characteristic for the subcritical region considered in Sect 5.2 .1 . 
Consider quantum numbers $|j|>k+1$. Then $g<g_{\mathrm{c}}(k+1)<g_{\mathrm{s}}(k+2) \leq g_{\mathrm{s}}(j)$, so that $g<g_{\mathrm{s}}(j)$. Such quantum numbers $j$ are characteristic for the nonsingular region considered in Sect 5.1 .

Therefore, we have

$$
\begin{aligned}
& U_{E}(\rho)= \begin{cases}U_{4 \nu, E}(\rho), & |j| \leq k, \\
U_{2 \nu, E}(\rho), & |j|=k+1, \\
U_{1 E}(\rho), & |j|>k+1,\end{cases} \\
& U_{n}(\rho)=\left\{\begin{array}{ll}
U_{4 \nu, n}(\rho), & |j| \leq k, \\
U_{2 \nu, n}(\rho), & |j|=k+1, \\
U_{1 n}(\rho), & |j|>k+1,
\end{array} \quad E_{n}= \begin{cases}E_{4 n}(\nu), & |j| \leq k, \\
E_{2 n}(\nu), & |j|=k+1, \\
E_{1 n}(Z, j, s), & |j|>k+1 .\end{cases} \right.
\end{aligned}
$$

C. Let $g \Longrightarrow(k, 3)$, that is, $g \in \Delta_{3}(k)$ for some $k$, which means that $g=g_{\mathrm{c}}(k+1)=k+1$.

Consider quantum numbers $|j| \leq k$. Then $g=g_{\mathrm{c}}(k+1)>g_{\mathrm{c}}(k) \geq g_{\mathrm{c}}(j)$, so that $g>g_{\mathrm{c}}(j)$. Such quantum numbers $j$ are characteristic for the overcritical region considered in Sect. 5.2.3.

Consider a quantum number $|j|=k+1$. Then $g=g_{\mathrm{c}}(j)$. Such quantum numbers $j$ are characteristic for the critical region considered in Sect. 5.2.2.

Consider quantum numbers $|j|>k+1$. Then $g=g_{\mathrm{c}}(k+1)<g_{\mathrm{s}}(k+2) \leq g_{\mathrm{s}}(j)$, so that $g<g_{\mathrm{s}}(j)$. Such quantum numbers $j$ are characteristic for the nonsingular region considered in Sect. 5.1 .

Therefore, for such charges, we have

$$
\begin{aligned}
& U_{E}(\rho)= \begin{cases}U_{4 \nu, E}(\rho), & |j| \leq k, \\
U_{3 \nu, E}(\rho), & |j|=k+1, \\
U_{1 E}(\rho), & |j|>k+1,\end{cases} \\
& U_{n}(\rho)=\left\{\begin{array}{ll}
U_{4 \nu, n}(\rho), & |j| \leq k, \\
U_{3 \nu, n}(\rho), & |j|=k+1, \\
U_{1 n}(\rho), & |j|>k+1,
\end{array} \quad E_{n}= \begin{cases}E_{4 n}(\nu), & |j| \leq k, \\
E_{3 n}(\nu), & |j|=k+1, \\
E_{1 n}(Z, j, s), & |j|>k+1 .\end{cases} \right.
\end{aligned}
$$

D. Let $g \in \Delta(0)$, which means that $g<g_{\mathrm{c}}( \pm 1 / 2)$. Consider quantum numbers $|j|>1 / 2$. Then

$$
g<g_{\mathrm{c}}\left( \pm \frac{1}{2}\right)=\frac{1}{2}<\sqrt{j^{2}-\frac{1}{4}}=g_{\mathrm{s}}(j)
$$

Such quantum numbers $j$ are characteristic for the nonsingular region considered in Sect 5.1 .

Consider quantum numbers $|j|=1 / 2$. Then $0=g_{\mathrm{s}}(j)<g<g_{\mathrm{c}}(j)=1 / 2$. Such quantum numbers $j$ are characteristic for the subcritical region considered in Sect 5.2.1. 
Therefore, for such charges, we have

$$
\begin{aligned}
& U_{E}(\rho)=\left\{\begin{array}{ll}
U_{2 \nu, E}(\rho), & |j|=1 / 2, \\
U_{1 E}(\rho), & |j|>1 / 2,
\end{array} \quad|E| \geq m,\right. \\
& U_{n}(\rho)=\left\{\begin{array}{ll}
U_{2 \nu, n}(\rho), & |j|=1 / 2, \\
U_{1 \nu, n}(\rho), & |j|>1 / 2,
\end{array} \quad E_{n}= \begin{cases}E_{2 n}(\nu), & |j|=1 / 2, \\
E_{1 n}(\nu), & |j|>1 / 2 .\end{cases} \right.
\end{aligned}
$$

E. Let $g=g_{\mathrm{c}}( \pm 1 / 2)$. Consider quantum numbers $|j|>1 / 2$. Then

$$
g=g_{\mathrm{c}}\left( \pm \frac{1}{2}\right)=\frac{1}{2}<\sqrt{j^{2}-\frac{1}{4}}=g_{\mathrm{s}}(j) .
$$

Such quantum numbers $j$ are characteristic for the nonsingular region considered in Sect. 5.1 .

Consider quantum numbers $|j|=1 / 2$. Then $g=g_{\mathrm{c}}(j)$. Such quantum numbers $j$ are characteristic for the critical region considered in Sect. 5.2 .2

Therefore, for such charges, we have

$$
\begin{aligned}
& U_{E}(\rho)=\left\{\begin{array}{ll}
U_{3 \nu, E}(\rho), & |j|=1 / 2, \\
U_{1 E}(\rho), & |j|>1 / 2,
\end{array} \quad|E| \geq m,\right. \\
& U_{n}(\rho)=\left\{\begin{array}{ll}
U_{3 \nu, n}(\rho), & |j|=1 / 2, \\
U_{1 \nu, n}(\rho), & |j|>1 / 2,
\end{array} \quad E_{n}= \begin{cases}E_{3 n}(\nu), & |j|=1 / 2, \\
E_{1 n}(\nu), & |j|>1 / 2 .\end{cases} \right.
\end{aligned}
$$

We are now in a position to describe the spectral problem for all the s.a. Dirac Hamiltonians with any coupling $g$. We note that the inequality $g>g_{\mathrm{s}}( \pm 1 / 2)=0$ and 10$)$ implies an important fact: the total s.a. Dirac Hamiltonian $\hat{H}_{s}$ is not uniquely determined for any charge $Z=(\epsilon g) / \alpha_{F}$.

Consider eigenvectors $\Psi_{s j}(\mathbf{r})$ of any s.a. Dirac Hamiltonian $\hat{H}_{s}^{\mathfrak{e}}$. They satisfy the following set of equations (see Sect. 3):

$$
\check{H}_{s} \Psi_{s j}(\mathbf{r})=E \Psi_{s j}(\mathbf{r}), \quad \hat{J}_{s} \Psi_{s j}(\mathbf{r})=j \Psi_{s j}(\mathbf{r}),
$$

where as the eigenvectors have the form $\Psi_{s j}(\mathbf{r})=V_{s j} U_{E}(\rho)$, see 77 .

For any coupling constants $g$, the energy spectrum of any s.a. Dirac Hamiltonian $\hat{H}_{s}^{\mathfrak{e}}$ contains the continuous spectrum occupying both negative and positive semiaxis $(-\infty,-m]$ and $[m, \infty)$ and of the discrete spectrum located in the interval $(0, m)$ and includes a growing infinite number energy levels, accumulated at the point $E=m$. Similar to the 3 -dimensional case (see. [13]), the asymptotic form as $n \rightarrow \infty$ of the spectrum is given by the well-known nonrelativistic formula

$$
E_{n}^{\text {nonrel }}=m-E_{n}=\frac{m g^{2}}{2 n^{2}} .
$$

The eigenfunctions $\Psi_{s j E}(\mathbf{r}),|E| \geq m$, which correspond to the continuous part of the spectrum, are generalized eigenfunctions of $\hat{H}_{s}^{\mathfrak{e}}$, whereas the eigenfunctions $\Psi_{s j E_{n}}(\mathbf{r})$ of $\hat{H}_{s}^{\mathfrak{e}}$, which correspond to the of bound states of 
energy $E_{n}$, belong to the Hilbert space $\mathfrak{H}$. Doublets $U_{E}(\rho)$ which correspond to the of bound states of energy $E_{n}$, are denoted by $U_{E_{n}}(\rho)=U_{n}(\rho)$. All the doublets $U_{E}(\rho)$ and $U_{n}(\rho)$ and the spectra $E_{n}$ depend on the extension parameters, on the quantum numbers $j$, parameter $s$ and on the coupling $g$ according to (44)-48). It should be remembered that the extension parameters depend on both $j$ and $s$.

\section{Spectral problem in regularized Coulomb field of the impurity}

In this section, we consider the bound state and point spectrum problems (for brevity, we speak about the point spectrum problem in what follows) for an electron in graphene with an impurity generating an electric field with a regularized Coulomb potential $V(\rho)$ given by

$$
V(\rho)=-g\left\{\begin{array}{cc}
1 / \rho_{0}, & \rho \leq \rho_{0}, \\
1 / \rho, & \rho \geq \rho_{0} .
\end{array}\right.
$$

Such a potential corresponds to the field of a positive charge impurity distributed uniformly over the spherical surface of the cutoff radius $\rho_{0}$ (Fig. 7). The parameter $g$ is defined in Eq. (2).

We assume that the impurity is placed in the middle of the hexagon of the graphene lattice. Regularization 49. represents a situation in which the lattice parameter $a$ is the closest distance that electron hopping between carbon sites can be from a potential source [8]. Then, we assume that the cutoff radius is of the order of the lattice constant $a$. For numerical calculations, we use the value $\rho_{0}=0.6 a$. The Dirac Hamiltonian $\hat{H}_{s}^{\text {reg }}$ is a



Figure 7: Regularized Coulomb potential.

differential operator in the Hilbert space $\mathfrak{H}=\mathbb{L}^{2}\left(\mathbb{R}^{2}\right)$, see Section 2 , associated with the differential operation

$$
\check{H}_{s}^{\mathrm{reg}}=-i\left(s \sigma_{x} \partial_{x}+\sigma_{y} \partial_{y}\right)+m \sigma_{z}+V(\rho)
$$

and naturally defined. Each Hamiltonian $\hat{H}_{s}(Z)$ with any $Z$ is a uniquely defined s.a. operator as the sum of the uniquely defined free Dirac Hamiltonian, see subsec. 5.1 and the bounded s.a. operator of multiplication by a bounded real-valued function $V(\rho)$. 
The Hamiltonian $\hat{H}_{s}^{\text {reg }}$ is rotationally invariant from the standpoint of the representation $U_{s}$ of the rotation group, and therefore, similarly to the point-like case, see Section 3 the point spectrum problem for $\hat{H}_{s}^{\text {reg }}$ is reduced to the point spectrum problem for partial radial Hamiltonians $\hat{h}_{\text {reg }}(Z, j, s)$ with fixed angular momenta $j= \pm 1 / 2,3 / 2, \ldots$ Each partial radial Hamiltonian $\hat{h}_{\mathrm{reg}}(Z, j, s)$ with fixed $Z, j, s$ is an s.a. operator in the Hilbert space $\mathbb{L}^{2}\left(\mathbb{R}_{+}\right)=L^{2}\left(\mathbb{R}_{+}\right) \oplus L^{2}\left(\mathbb{R}_{+}\right)$of doublets $F(\rho)=(f(\rho), g(\rho))^{T}(f(\rho)$ and $g(\rho)$ are called the radial functions) associated with the partial radial differential operation

$$
\check{h}_{\mathrm{reg}}(Z, j, s)=-i \sigma_{y} \frac{d}{d \rho}+\frac{\kappa}{\rho} \sigma_{x}+V(\rho)+m \sigma_{z},
$$

and defined on the natural domain for $\breve{h}$ : the domain $D_{h}$ of each $\hat{h}$ consists of doublets $F(\rho)$ that are absolutely continuous on $(0, \infty)$, vanish at the origin, $f(0)=g(0)=0$, and are square integrable together with $\check{h} F(\rho)$ on $(0, \infty)$.

Because the potential $V(\rho)$ vanishes at infinity, the spectrum of each partial radial Hamiltonian $\hat{h}_{\text {reg }}(Z, j, s)$ consists of a continuous part that is the union $(-\infty,-m] \cup[m, \infty)$ of two half-axis and a point spectrum $\left\{E_{n}(Z, j, s), n \in \mathbb{Z}_{+}\right\}$located in the segment $[-m, m]$. The total point spectrum p.spec $\hat{H}_{s}^{\mathrm{reg}}(Z)$ of the Dirac Hamiltonian $\hat{H}_{s}^{\mathrm{reg}}(Z)$, which is the subject of our main interest, is a union of the point spectra of partial radial hamiltonians $\hat{h}_{\mathrm{reg}}(Z, j, s)$,

$$
\text { p.spec } \hat{H}_{s}^{\mathrm{reg}}(Z)=\cup_{j} \text { p.spec } \hat{h}_{\mathrm{reg}}(Z, j, s) \text {, }
$$

while the corresponding eigenfunctions $\Psi_{s j}(\mathbf{r})$ of $\hat{H}_{s}(Z)$ in $\mathbb{L}^{2}\left(\mathbb{R}^{2}\right)$ are obtained from the eigenfunctions $F(\rho)$ of $\hat{h}_{\text {reg }}(Z, j, s)$ in $\mathbb{L}^{2}\left(\mathbb{R}_{+}\right)$by the unitary transformation $V_{s j}$, see 7 .

The point spectrum and the corresponding eigenfunctions of a partial radial Hamiltonian $\hat{h}_{\text {reg }}(Z, j, s)$ are defined by the solutions of the stationary partial radial Schrödinger equation

$$
\hat{h}_{\mathrm{reg}}(Z, j, s) F(\rho)=E F(\rho), \quad E \in[-m, m], \quad F(\rho) \in D_{h(Z, j, s)} .
$$

This equation with fixed $Z, j$, and $s$ implies the system of differential radial equations for the radial functions $f(\rho)$ and $g(\rho)$ :

$$
\begin{aligned}
& f^{\prime}(\rho)+\frac{\kappa}{\rho} f(\rho)-k_{+}(\rho) g(\rho)=0, \\
& g^{\prime}(\rho)-\frac{\kappa}{\rho} g(\rho)+k_{-}(\rho) f(\rho)=0, \quad k_{ \pm}(\rho)=E-V(\rho) \pm m .
\end{aligned}
$$

The system of radial equations (50) is supplemented by the condition $-m \leq E \leq m$ and the following conditions on the functions $f(\rho)$ and $g(\rho)$ : they are absolutely continuous in $\rho$ on $(0, \infty)$, satisfy the zero boundary conditions at the origin, $f(0)=g(0)=0$, and are square integrable on $(0, \infty)$ (in fact, at infinity).

In finding the point spectra $\left\{E_{n}(Z, j, s)\right\}$ within the segment $-m \leq E \leq m$, we must consider the open energy interval $-m<E<m$ and its endpoints $E=m$ and $E=-m$ separately for technical reasons that become clear below. We begin with the energy region $-m<E<m$. 


\section{Discrete spectrum in $(-m, m)$}

\subsection{Solution of radial equations for $0 \leq \rho \leq \rho_{0}$}

In the interior region $0 \leq \rho \leq \rho_{0}$, where we set $f(\rho)=f_{\text {in }}(\rho)$ and $g(\rho)=g_{\text {in }}(\rho)$, the functions $k_{ \pm}(\rho)$ in Eqs. (50) and 49) are constants,

$$
\begin{aligned}
& k_{+}(\rho)=k_{+}=E+\frac{g}{\rho_{0}}+m, \\
& k_{-}(\rho)=k_{-}=E+\frac{g}{\rho_{0}}-m, \quad E \in(-m, m) .
\end{aligned}
$$

In this region, the first equation in 50 can be rewritten as

$$
g_{\text {in }}(\rho)=\frac{1}{k_{+}}\left[f_{i n}^{\prime}(\rho)+\frac{\kappa}{\rho} f_{i n}(\rho)\right] .
$$

Then, the second equation in 50 yields the second-order differential equation for the function $f_{i n}(\rho)$,

$$
\begin{gathered}
\frac{d^{2} f_{\text {in }}(\rho)}{d \rho^{2}}+\left(\eta^{2}-\frac{\nu^{2}-1 / 4}{\rho^{2}}\right) f_{\text {in }}(\rho)=0, \nu \in \mathbb{Z}_{+}, \\
\eta=\sqrt{k_{+} k_{-}}, \quad k_{+} k_{-}=\left(E+\frac{g}{\rho_{0}}\right)^{2}-m^{2}, \quad \nu=\left|\kappa+\frac{1}{2}\right|=|j|+\frac{\zeta}{2}=\left\{\begin{array}{c}
|j|+1 / 2, \zeta=1, \\
|j|-1 / 2, \zeta=-1 .
\end{array}\right.
\end{gathered}
$$

Equation (51) is supplemented by the two boundary conditions at the origin:

$$
f_{\text {in }}(0)=0,\left.\left[f_{\text {in }}^{\prime}(\rho)+\frac{\kappa}{\rho} f_{\text {in }}(\rho)\right]\right|_{\rho=0}=0
$$

the latter is due to the condition $g_{\text {in }}(0)=0$. Note that the second condition is nontrivial only in the case $\nu=0$, if $\nu \neq 0$, it follows from the first one.

The general solution of Eq. [51] under the above boundary conditions is (see [22])

$$
f_{\text {in }}(\rho)=c \sqrt{\rho} J_{\nu}(\eta \rho)=c \sqrt{\rho}\left\{\begin{array}{l}
J_{|j|+1 / 2}(\eta \rho), \quad \zeta=1, \\
J_{|j|-1 / 2}(\eta \rho), \zeta=-1,
\end{array} \quad c \in \mathbb{C} .\right.
$$

Using the relation $J_{\nu}^{\prime}(z) \mp(\nu / z) J_{\nu}(z)=\mp J_{\nu \pm 1}(z)$, see [23], we find

$$
g_{\text {in }}(\rho)=c \sqrt{\rho} \sqrt{\frac{k_{-}}{k_{+}}} \zeta J_{\nu-\zeta}(\eta \rho)=c \sqrt{\rho} \sqrt{\frac{k_{-}}{k_{+}}}\left\{\begin{array}{l}
J_{|j|-1 / 2}(\eta \rho), \quad \zeta=1, \\
-J_{|j|+1 / 2}(\eta \rho), \quad \zeta=-1 .
\end{array}\right.
$$

\subsection{Solution of radial equations for $\rho_{0} \leq \rho<\infty$}

In the exterior region $\rho \in\left[\rho_{0}, \infty\right)$, where we set $f(\rho)=f_{\text {out }}(\rho), g(\rho)=g_{\text {out }}(\rho)$, system (50), 49) is identical in form with the system of equations in the 3-dimensional Coulomb problem with a point charge, see [13] and [16], the only difference is that the charge parameter $g=\alpha_{F} Z / \epsilon$ is replaced by $\alpha Z, \alpha$ is the fine structure constant, and the parameter $\kappa=-s j, s= \pm 1, j= \pm 1 / 2, \pm 3 / 2, \ldots$, is replaced by $\varkappa=\zeta(j+1 / 2), \zeta= \pm 1$, 
$j=1 / 2,3,2, \ldots$. The general solution of the latter system is well known. In our case, system 50 and 49 is supplemented by the condition that the functions $f_{\text {out }}(\rho)$ and $g_{\text {out }}(\rho)$ are square integrable at infinity. Under this condition, a solution of the system is given by:

$$
\begin{aligned}
& f_{\text {out }}(\rho)=B \sqrt{\frac{2 m}{m-E}}(2 \beta \rho)^{\mu} e^{-\beta \rho}\left[b_{-} \Psi(a+1, c ; 2 \beta \rho)+\Psi(a, c ; 2 \beta \rho)\right], \\
& g_{\text {out }}(\rho)=B \sqrt{\frac{2 m}{m+E}}(2 \beta \rho)^{\mu} e^{-\beta \rho}\left[b_{-} \Psi(a+1, c ; 2 \beta \rho)-\Psi(a, c ; 2 \beta \rho)\right],
\end{aligned}
$$

where

$$
\beta=\sqrt{m^{2}-E^{2}}, \quad \mu=\sqrt{\kappa^{2}-g^{2}}, \quad a=\mu-\frac{g E}{\beta}, \quad c=1+2 \mu, \quad b_{-}=\kappa+\frac{g m}{\beta},
$$

and $\Psi$ is a standard confluent hypergeometric function vanishing at infinity, see (14).

In what follows, we use Whittaker functions, see [23,

$$
W_{\lambda, \mu}(x)=e^{-x / 2} x^{c / 2} \Psi(a, c ; x), \lambda=\frac{c}{2}-a, \mu=\frac{c-1}{2},
$$

and a new energy variable $\varepsilon$ defined as $E=m \cos \varepsilon, \varepsilon=\arccos (E / m) \in(0, \pi)$. In these terms, the final form of solutions of Eqs. 50 and 49 in the exterior region $\rho_{0} \leq \rho<\infty$ reads:

$$
\begin{aligned}
& f_{\text {out }}(\rho)=B \csc \left(\frac{\varepsilon}{2}\right)(2 \beta \rho)^{-1 / 2}\left[(g \csc \varepsilon+\kappa) W_{\lambda^{\prime}, \mu}(2 \beta \rho)+W_{\lambda, \mu}(2 \beta \rho)\right], \\
& g_{\text {out }}(\rho)=B \sec \left(\frac{\varepsilon}{2}\right)(2 \beta \rho)^{-1 / 2}\left[(g \csc \varepsilon+\kappa) W_{\lambda^{\prime}, \mu}(2 \beta \rho)-W_{\lambda, \mu}(2 \beta \rho)\right],
\end{aligned}
$$

where

$$
\beta=m \sin \varepsilon, \quad \lambda=\frac{g E}{\beta}+\frac{1}{2}=g \cot \varepsilon+\frac{1}{2}, \quad \lambda^{\prime}=\lambda-1=g \cot \varepsilon-\frac{1}{2} .
$$

\subsection{Numerical solutions for discrete spectrum in the region $(-m, m)$,}

After the general solution of Eqs. (50) is found in the respective regions $0 \leq \rho \leq \rho_{0}$ and $\rho_{0} \leq \rho<\infty$, it remains to satisfy the basic continuity condition for the solution as a whole (to sew the partial solutions together smoothly), which reduces to the requirement of continuity of the solution at the point $\rho=\rho_{0}$ :

$$
f_{\text {in }}\left(\rho_{0}\right)=f_{\text {out }}\left(\rho_{0}\right), \quad g_{\text {in }}\left(\rho_{0}\right)=g_{\text {out }}\left(\rho_{0}\right) .
$$

If $c$ and $B$ are not zero, the compatibility of these conditions yields the transcendental equation, which determines the point energy spectrum in the region $(-m, m)$, which appears to be a discrete one in terms of the variable $\varepsilon$,

$$
\begin{aligned}
& J_{\nu}\left(\eta \rho_{0}\right) \sec \left(\frac{\varepsilon}{2}\right)\left[(\kappa+g \csc \varepsilon) W_{\lambda^{\prime}, \mu}\left(2 \beta \rho_{0}\right)-W_{\lambda, \mu}\left(2 \beta \rho_{0}\right)\right] \\
& -\sqrt{\frac{k_{-}}{k_{+}}} \zeta J_{\nu-\zeta}\left(\eta \rho_{0}\right) \csc \left(\frac{\varepsilon}{2}\right)\left[(\kappa+g \csc \varepsilon) W_{\lambda^{\prime}, \mu}\left(2 \beta \rho_{0}\right)+W_{\lambda, \mu}\left(2 \beta \rho_{0}\right)\right]=0,
\end{aligned}
$$


with $k_{ \pm}=m(\cos \varepsilon \pm 1)+g / \rho_{0}$. We call this basic equation the spectrum equation for the interval $(-m, m)$. Strictly speaking, we deal with a series of exact spectrum equations for given $Z, j$ and $s$.

After the spectrum equation is solved, i.e., the energy eigenvalues $E_{n}(Z, j, s)$ of the partial radial Hamiltonians $\hat{h}_{\text {reg }}(Z, j, s)$ are found, the corresponding eigenfunctions $F_{n}(Z, j, s) \in \mathbb{L}^{2}\left(\mathbb{R}_{+}\right)$of bound states of Hamiltonians $\hat{h}_{\text {reg }}(Z, j, s)$ are obtained by the substitution of the evaluated energy eigenvalues $\left.E_{n}(Z, j, s)\right)$ for $E$ in formulas (52), (53), and (54) with due regard to continuity condition (56), according to which a unique normalization constant for wave functions (doublets) remains undetermined. An analytical solution of the spectrum equation (57) with any $Z, j, s$ is beyond the scope of our possibilities. Only numerical solution of these equations is realizable at present.

An equivalent expanded form of the spectrum equation (57) with the certain fixed $\zeta$, which is maybe more suitable for numerical calculations, is

$$
\sqrt{\frac{k_{-}}{k_{+}}} \frac{J_{|j|-1 / 2}\left(\eta \rho_{0}\right)}{J_{|j|+1 / 2}\left(\eta \rho_{0}\right)}-\tan \left(\frac{\varepsilon}{2}\right) \frac{\left[(|j|+g \csc \varepsilon) W_{\lambda^{\prime}, \mu}\left(2 \beta \rho_{0}\right)-W_{\lambda, \mu}\left(2 \beta \rho_{0}\right)\right]}{\left[(|j|+g \csc \varepsilon) W_{\lambda^{\prime}, \mu}\left(2 \beta \rho_{0}\right)+W_{\lambda, \mu}\left(2 \beta \rho_{0}\right)\right]}=0,
$$

for $\zeta=1$ and

$$
\sqrt{\frac{k_{-}}{k_{+}}} \frac{J_{|j|+1 / 2}\left(\eta \rho_{0}\right)}{J_{|j|-1 / 2}\left(\eta \rho_{0}\right)}+\tan \left(\frac{\varepsilon}{2}\right) \frac{\left[(-|j|+g \csc \varepsilon) W_{\lambda^{\prime}, \mu}\left(2 \beta \rho_{0}\right)-W_{\lambda, \mu}\left(2 \beta \rho_{0}\right)\right]}{\left[(-|j|+g \csc \varepsilon) W_{\lambda^{\prime}, \mu}\left(2 \beta \rho_{0}\right)+W_{\lambda, \mu}\left(2 \beta \rho_{0}\right)\right]}=0,
$$

for $\zeta=-1$.

Numerical calculations for special case $j=1 / 2, s=1$, which produces the lowest energy levels, are shown in Fig. 8.

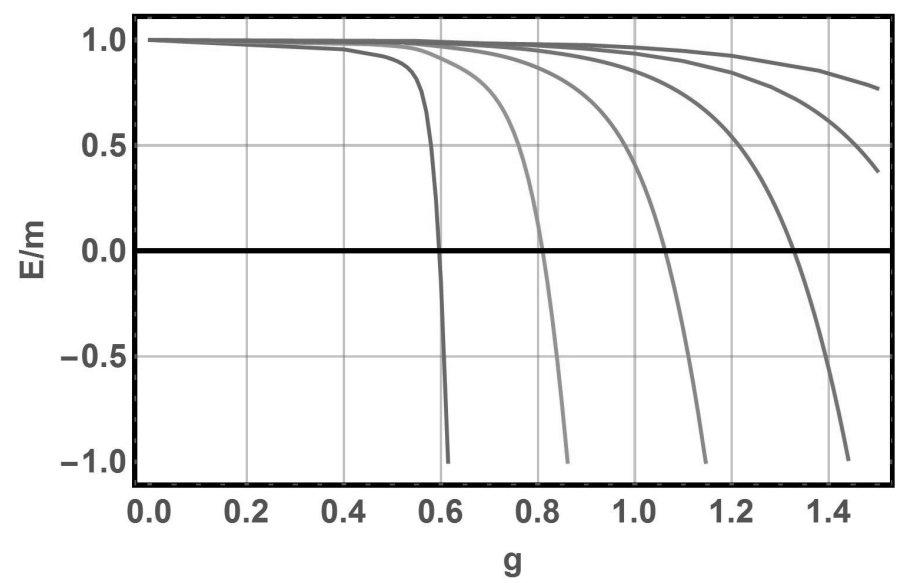

Figure 8: The $g$ dependence of the lowest energy levels for $j=1 / 2, s=1$. 


\section{$9 \quad$ Eigenstates for $E= \pm m$}

The preceding consideration fails for the points $E=m$ and $E=-m$ because according to Eqs. (54) and (55) at these points the variable $\beta=\sqrt{m^{2}-E^{2}}$ becomes zero, while the factors $1 / \sqrt{m-E}, 1 / \sqrt{m+E}, b_{-}=\kappa+g m / \beta$, and the parameter $a=\mu-g E / \beta$ blow up. Therefore, these points have to be considered separately.

\subsection{The point $E=m$}

It suffices to examine the set 50 and 49 with $E=m$ in the exterior region $\rho_{0} \leq \rho<\infty$ where it has the form

$$
\begin{aligned}
& f^{\prime}(\rho)+\frac{\kappa}{\rho} f(\rho)-\left(2 m+\frac{g}{\rho}\right) g(\rho)=0, \\
& g^{\prime}(\rho)-\frac{\kappa}{\rho} g(\rho)+\frac{g}{\rho} f(\rho)=0 .
\end{aligned}
$$

It has to be supplemented by conditions that the both $f(\rho)$ and $g(\rho)$ functions are absolutely continuous and square integrable together with their derivatives on $\left(\rho_{0}, \infty\right)$.

Using second equation (58), we find that the function $g(\rho)$ satisfies the second-order differential equation

$$
g^{\prime \prime}(\rho)+\frac{1}{\rho} g^{\prime}(\rho)+\left(\frac{2 g m}{\rho}-\frac{\kappa^{2}-g^{2}}{\rho^{2}}\right) g(\rho)=0 .
$$

The substitution $g(\rho)=w(z), z=2 \sqrt{2 g m \rho}$ reduces Eq. 59 to the Bessel equation,

$$
w^{\prime \prime}(z)+\frac{1}{z} w^{\prime}(z)+\left(1-\frac{\tilde{\nu}^{2}}{z^{2}}\right) w(z)=0
$$

with $\tilde{\nu}=2 \mu=2 \sqrt{j^{2}-g^{2}}, 2 \sqrt{2 g m \rho_{0}} \leq z<\infty$. The general solution of this equation is given by $w(z)=$ $c_{1} H_{\tilde{\nu}}^{(1)}(z)+c_{2} H_{\tilde{\nu}}^{(2)}(z)$, where $H_{\nu}^{(1)}(z)$ and $H_{\nu}^{(2)}(z)$ are the respective first and second Hankel functions, see [23]. Its asymptotic behavior at infinity is given by $(z \rightarrow \infty)$

$$
\begin{aligned}
w(z)= & c_{1} \sqrt{\frac{2}{\pi z}} \exp \left[\frac{i}{4}(4 z-2 \pi \tilde{\nu}-\pi)\right]\left[1+O\left(\frac{1}{z}\right)\right] \\
& +c_{2} \sqrt{\frac{2}{\pi z}} \exp \left[-\frac{i}{4}(4 z-2 \pi \tilde{\nu}-\pi)\right]\left[1+O\left(\frac{1}{z}\right)\right] .
\end{aligned}
$$

In view of second equation (58), it follows that the asymptotic behavior of both functions $f(\rho)$ and $g(\rho)$ at infinity is estimated as $f(\rho), g(\rho)=O\left(\rho^{-1 / 4}\right), \rho \rightarrow \infty$, so that the both functions are not square-integrable at infinity. This means that system 58 has no square-integrable solutions and therefore, there are no bound states with the energy $E=m$, i.e., with zero binding energy, for an electron in the Coulomb field of any charge $Z$ with cutoff 49 , as well as in the Coulomb field of a point charge. 


\subsection{The point $E=-m$}

The system (50) of radial equations for bound states with $E=-m$, i.e. with binding energy $2 m$, becomes

$$
\begin{aligned}
& f^{\prime}(\rho)+\frac{\kappa}{\rho} f(\rho)+V(\rho) g(\rho)=0 \\
& g^{\prime}(\rho)-\frac{\kappa}{\rho} g(\rho)-[V(\rho)+2 m] f(\rho)=0
\end{aligned}
$$

being supplemented by the conditions that the both $f(\rho)$ and $g(\rho)$ are absolutely continuous together with their first derivatives and square integrable on $(0, \infty)$ and become zero at the origin, $f(0)=0, g(0)=0$.

\subsubsection{Solutions in the region $0 \leq \rho \leq \rho_{0}$}

The general solution of system 60 and 490 in the interior region $0 \leq \rho \leq \rho_{0}$, where we set $f(\rho)=f_{\text {in }}(\rho)$ and $g(\rho)=g_{\text {in }}(\rho)$, under the above conditions is given by

$$
\begin{aligned}
& f_{\text {in }}(\rho)=c \sqrt{\rho} J_{\nu}\left(\eta_{0} \rho\right)=c \sqrt{\rho}\left\{\begin{array}{l}
J_{|j|+1 / 2}\left(\eta_{0} \rho\right), \quad \zeta=1, \\
J_{|j|-1 / 2}\left(\eta_{0} \rho\right), \zeta=-1,
\end{array}\right. \\
& g_{\text {in }}(r)=c \sqrt{\rho} \sqrt{1-\frac{2 m \rho_{0}}{g}} \zeta J_{\nu-\zeta}\left(\eta_{0} \rho\right)=c \sqrt{\rho} \sqrt{1-\frac{2 m \rho_{0}}{g}}\left\{\begin{array}{l}
J_{|j|-1 / 2}\left(\eta_{0} \rho\right), \zeta=1, \\
-J_{|j|+1 / 2}\left(\eta_{0} \rho\right), \zeta=-1,
\end{array}\right.
\end{aligned}
$$

where $\eta_{0}=\left(g / \rho_{0}\right) \sqrt{1-2 m \rho_{0} / g}$. It suffices to set $E=-m$ in 51, , 52, and 53.

\subsubsection{Solutions in the region $\rho_{0} \leq \rho<\infty$}

In the exterior region $\rho_{0} \leq \rho<\infty$, where we set $f(\rho)=f_{\text {out }}(\rho)$ and $g(\rho)=g_{\text {out }}(\rho)$, Eqs. 60] and 496) become

$$
\begin{aligned}
& f_{\text {out }}^{\prime}(\rho)+\frac{\kappa}{\rho} f_{\text {out }}(\rho)-\frac{g}{\rho} g_{\text {out }}(\rho)=0 \\
& g_{\text {out }}^{\prime}(\rho)-\frac{\kappa}{\rho} g_{\text {out }}(\rho)-\left(2 m-\frac{g}{\rho}\right) f_{\text {out }}(\rho)=0
\end{aligned}
$$

under conditions that both functions $f_{\text {out }}(\rho)$ and $g_{\text {out }}(\rho)$ are absolutely continuous and square-integrable together with their derivatives on $\left(\rho_{0}, \infty\right)$.

Using first Eq. 61] in the second one, we find

$$
f_{\text {out }}^{\prime \prime}(\rho)+\frac{1}{\rho} f_{\text {out }}^{\prime}(\rho)-\frac{2 g m}{\rho} f_{\text {out }}(\rho)-\frac{\kappa^{2}-g^{2}}{\rho^{2}} f_{\text {out }}(\rho)=0 .
$$

The substitution $f_{\text {out }}(\rho)=w(z), z=2 \sqrt{2 g m \rho}$, reduces Eq. 62 to the one for the modified Bessel functions, see [23],

$$
w^{\prime \prime}(z)+\frac{1}{z} w^{\prime}(z)-\left(1+\frac{\tilde{\nu}^{2}}{z^{2}}\right) w(z)=0, \quad \tilde{\nu}=2 \mu=2 \sqrt{j^{2}-g^{2}}, \quad 2 \sqrt{2 g m \rho_{0}} \leq z<\infty .
$$


The requirement for $f_{\text {out }}(\rho)$ to be square-integrable at infinity then yields $f_{\text {out }}(\rho)=A K_{\tilde{\nu}}(z)$, where $K_{\tilde{\nu}}(z)$ is the McDonald function,

$$
\begin{aligned}
& K_{\tilde{\nu}}(z)=\frac{\pi}{2 \sin \pi \tilde{\nu}}\left[I_{-\tilde{\nu}}(z)-I_{\tilde{\nu}}(z)\right], \quad \tilde{\nu} \neq n \in \mathbb{Z}_{+}, \\
& I_{\tilde{\nu}}(z)=\sum_{m=0}^{\infty} \frac{(z / 2)^{2 m+\tilde{\nu}}}{m ! \Gamma(m+\tilde{\nu}+1)}, \quad K_{\tilde{\nu}}(z)=K_{-\tilde{\nu}}(z) .
\end{aligned}
$$

For $\tilde{\nu}=n \in \mathbb{Z}_{+}$, the functions $K_{n}(z)$ contain terms with a logarithmic factor, see [23].

We finally obtain a general solution of Eqs. 61):

$$
\begin{aligned}
f_{\text {out }}(\rho) & =A K_{\tilde{\nu}}(z), \quad z=2 \sqrt{2 g m \rho}, \\
g_{\text {out }}(\rho) & =\frac{A}{g}\left[\frac{z}{2} \frac{d}{d z} K_{\tilde{\nu}}(z)+\kappa K_{\tilde{\nu}}(z)\right]=\frac{A}{g}\left\{-\frac{z}{4}\left[K_{\tilde{\nu}-1}(z)+K_{\tilde{\nu}+1}(z)\right]+\kappa K_{\tilde{\nu}}(z)\right\},
\end{aligned}
$$

where we have used the known formula $K_{\tilde{\nu}-1}(z)+K_{\tilde{\nu}+1}(z)=-2 K_{\tilde{\nu}}^{\prime}(z)$ (see [23]).

\section{Supercritical charges}

After the general solution of system 60 is found independently in the respective regions $0 \leq \rho \leq \rho_{0}$ and $\rho_{0} \leq \rho<\infty$, it remains to satisfy the basic continuity condition for the solution as a whole (to sew the partial solutions together smoothly), which reduces to the requirement of continuity of the solution at the point $\rho=\rho_{0}$,

$$
f_{\text {in }}\left(\rho_{0}\right)=f_{\text {out }}\left(\rho_{0}\right), \quad g_{\text {in }}\left(\rho_{0}\right)=g_{\text {out }}\left(\rho_{0}\right) .
$$

The compatibility of equalities 63 with $c \neq 0, A \neq 0$ yields the relation

$$
\begin{gathered}
J_{\nu}\left(\eta_{0} \rho_{0}\right)\left\{-\frac{z_{0}}{4}\left[K_{\tilde{\nu}-1}\left(z_{0}\right)+K_{\tilde{\nu}+1}\left(z_{0}\right)\right]+\kappa K_{\tilde{\nu}}\left(z_{0}\right)\right\}-\left(\eta_{0} \rho_{0}\right) \zeta J_{\nu-\zeta}\left(\eta_{0} \rho_{0}\right) K_{\tilde{\nu}}\left(z_{0}\right)=0, \\
\eta_{0} \rho_{0}=g \sqrt{1-\frac{2 m \rho_{0}}{g}}, \quad z_{0}=2 \sqrt{2 g m \rho_{0}},
\end{gathered}
$$

that can be considered as an equation for coupling constants $g$ that provides bound states with the energy $E=-m$. We let $g^{(-m)}(j, s)$ denote such coupling constants.

An analytical solution of equation 64 for $g^{(-m)}(j, s)$ with arbitrary $j, s$ is unlikely to be possible at present. We only can try to analyze it qualitatively and solve it numerically.

An equivalent form of equation (64) that is more suitable for its qualitative analysis and its numerical solution reads

$$
\left(\eta_{0} \rho_{0}\right) \frac{\zeta J_{\nu-\zeta}\left(\eta_{0} \rho_{0}\right)}{J_{\nu}\left(\eta_{0} \rho_{0}\right)}+\left[\frac{z_{0}}{4} \frac{K_{\tilde{\nu}-1}\left(z_{0}\right)+K_{\tilde{\nu}+1}\left(z_{0}\right)}{K_{\tilde{\nu}}\left(z_{0}\right)}-\kappa\right]=0
$$

There exists an infinitely growing sequence $\left\{g_{n}^{(-m)}(j, s), n \in \mathbb{N}\right\}, g_{n}^{(-m)}(j, s) \rightarrow \infty$ as $n \rightarrow \infty$, of roots of this equation with any fixed $j, s,[16$. This infinite sequence yields the corresponding infinitely growing sequence $\left\{Z_{n}^{(-m)}(j, s)=\alpha_{F}^{-1} \epsilon g_{n}^{(-m)}(j, s), n \in \mathbb{N}\right\}$ of charges $Z$. 




Figure 9: Graphic solution of Eq. 65.

We now define the supercritical charge $Z_{\text {scr }}(j, s)$ for each pair of quantum numbers $j, s$ as an integer nearest to $Z_{1}(j, s)$ (the first term in the sequence $\left\{Z_{n}^{(-m)}(j, s), n \in \mathbb{N}\right\}$ ) from above,

$$
Z_{\mathrm{scr}}(j, s)=\left\{\begin{array}{l}
{\left[Z_{1}(j, s)\right]+1, Z_{1}(j, s) \notin \mathbb{N}, \quad Z_{1}(j, s)=\left(\alpha_{F}^{-1} \epsilon\right) g_{1}^{(-m)}(j, s),} \\
Z_{1}(j, s), Z_{1}(j, s) \in \mathbb{N},
\end{array}\right.
$$

the symbol $[\ldots]$ denotes the integral part of a real number.

We believe that the supercritical charge is defined by the minimum of all the charges $Z_{n}^{(-m)}(j, s)$, which is attained in the sector $j=1 / 2, s=1$ and is equal to

$$
Z_{\text {scr }}=\left\{\begin{array}{l}
{\left[Z_{1}(1 / 2,1)\right]+1, Z_{1}(1 / 2,1) \notin \mathbb{N}} \\
Z_{1}(1 / 2,1), Z_{1}(1 / 2,1) \in \mathbb{N}
\end{array}\right.
$$

The corresponding coupling $g_{\mathrm{scr}}=g_{1}^{(-m)}(1 / 2,1)$ also can be called the supercritical coupling.

It is worth noting that the supercritical charges depend on a regularization of the Coulomb field, in other words, the supercritical charges are model dependent, and in addition, they depend on the parameters $\alpha_{F}$ and $\epsilon$.

To determine $g_{n}^{(-m)}(1 / 2,1)$, it is convenient to represent Eq. 65 as $\phi(g)=0$,

$$
\phi(g)=\left(\eta_{0} \rho_{0}\right) \frac{J_{1}\left(\eta_{0} \rho_{0}\right)}{J_{0}\left(\eta_{0} \rho_{0}\right)}-\left[\frac{z_{0}}{4} \frac{\left[K_{\tilde{\nu}-1}\left(z_{0}\right)+K_{\tilde{\nu}+1}\left(z_{0}\right)\right]}{K_{\tilde{\nu}}\left(z_{0}\right)}+\frac{1}{2}\right],
$$

where $\tilde{\nu}=\sqrt{1-4 g^{2}}$.

Results of numerical calculations are presented graphically in Fig. 9, the first lower values of $g_{n}^{(-m)}(1 / 2,1)$ are:

$$
\left\{g_{n}^{(-m)}(1 / 2,1)\right\}=\{0.54731,0.767737,1.05737,1.35964,1.65711, \ldots\},
$$

for $m=0.26 \mathrm{eV}$. Sequence 66 corresponds to the $\left\{Z_{n}^{(-m)}(1 / 2,1)\right\}$. Then, we have $g_{\text {scr }}=g_{1}^{(-m)}(1 / 2,1)=$ 0.54731 . 


\section{Conclusion}

Solving spectral problems for electronic excitations in a graphene in the presence of point-like Coulomb impurities, we have demonstrated that from the mathematical standpoint, there is no problem in defining s.a. Hamiltonians that define energy spectra and the corresponding complete sets of eigenfunctions for any charge of the impurities. We have constructed families of such possible s.a. Hamiltonians that are parameterized by some extension parameters. The general theory thus describes all the possibilities that can be offered to a physicist for his choice. This choice is a completely physical problem.

Energy levels were calculated and corresponding (generalized) eigenfunctions were obtained for any charge of the impurities (see Eqs. 444-48) ). Namely, for the nonsingular region $\left(g \leq g_{\mathrm{s}}(j)\right)$ energy levels and normalized (generalized) eigenfunctions were described by Eqs. 26) and (27), 28) respectively. The same was done for the subcritical region $\left(g_{\mathrm{s}}(j)<g<g_{\mathrm{c}}(j)\right)$, for the critical region $\left(g=g_{\mathrm{c}}(j)\right)$, and for the overcritical region $\left(g>g_{\mathrm{c}}(j)\right)$, see Eqs. $30-31,37-38$ and $40-41$ respectively.

We stress that the obtained eigenfunctions can be used to calculate a local density of states, which can be measured experimentally by using the scanning tunneling microscopy [24]. The importance of calculations in the graphene with Coulomb impurities is confirmed by results of the work [9], where it was shown that, in contrast to an undoped graphene, there are significant differences in the behavior of a local density of states near the boundary of the positive continuum.

It is interesting to note that in our problem, the critical coupling $g_{\mathrm{c}}( \pm 1 / 2)=1 / 2$ and the lower critical coupling $g_{\mathrm{s}}( \pm 1 / 2)=0$, whereas, in the 3 -dimensional case they are $g_{\mathrm{c}}(1 / 2)=1, g_{\mathrm{s}}(1 / 2)=\sqrt{3} / 2$. Besides, due to the large value of the fine structure constant in graphene, $\alpha_{F} \simeq 2.2$, the critical value of the impurity charge is small: $Z_{\mathrm{c}}=Z_{\mathrm{c}}( \pm 1 / 2) \simeq 1$, which opens the possibility of testing the supercritical instability in the graphene [7]. Indeed, the supercritical atomic collapse in graphene was observed experimentally and reported in Ref. [25].

In contrast to the 3 -dimensional case, in the problem under consideration for all values of impurity charge corresponding s.a. Hamiltonians are not defined uniquely. We recall that the Dirac Hamiltonian for an electron in the Coulomb field in 3 dimensions is defined uniquely for $Z \leq 118$. We note that a transition through critical charges does not lead to any technical qualitative changes in the mathematical description.

It should be noted that in the Ref. [26] a bound-state spectrum of low-energy excitations in a gapped graphene with a charged impurity, was studied without a convinced analysis of the asymptotic behavior of wave functions based on a correct construction of a corresponding s.a. Dirac Hamiltonian. Besides, in the Ref. [27] s.a. Dirac Hamiltonians with the Coulomb field in combination with the Aharonov-Bohm field in $2+1$ dimensions and their spectral analysis were considered. However, in this consideration, features of the graphene problem were not taken into account. Because of this, the radial Hamiltonians which were considered in this work, are parametrized in a specific way which does not allow one to identify them with the corresponding Hamiltonians of real graphene problem. Moreover, the zero limit of the additional external field (the Aharonov-Bohm field), 
which is necessary for possible comparison, was not studied and seems like is a nontrivial task.

Studying the spectral problem in the graphene with impurities presented by a regularized Coulomb potential (see Section 7), we have found the so-called supercritical charges, for which lowest levels of energy spectra reach the value $-m$. Formally (see discussion in Ref. [16, where the corresponding $3+1$ dimensional case was studied, and Refs. [28, 29]), this may be an indication that for such charges the vacuum becomes unstable with respect to possible pair creation. At the same time this may be an indication that the problem becomes many particle one, such that one-particle relativistic quantum mechanics based on the Dirac Hamiltonian fails. Then, calculations in the framework of the latter model may be not sufficient for statements about the existence of real physical effects such as particle production. For this reason, we believe that the problem of the production of electron-positron pairs from the vacuum by a supercritical Coulomb field is still far from its completion. That is why we cannot accept the conclusion of the work [15] that a real production of electron-positron pairs by a regularized supercritical Coulomb field is impossible.

Note that the Eq. (65) that defines the supercritical coupling constant $g_{\text {scr }}$ has solutions only for $g_{\text {scr }}>$ $g_{\mathrm{c}}( \pm 1 / 2)=0.5$. For $\rho_{0}=0.6 a$ and $m=0.26 \mathrm{eV}$, we obtain $g_{\mathrm{scr}}=0.54731$, which corresponds to small values $Z_{\mathrm{scr}} \simeq Z_{\mathrm{c}} \simeq 1$ for the dielectric constant in the range $\epsilon \approx 2.4-5$. Thus, even after the regularization, the supercritical charge is the same as critical charge for the point-like Coulomb field case. We note that in 3dimensional case, for the regularized Coulomb potential the supercritical charge $Z_{\text {scr }}=174$, is greater than the corresponding critical charge $Z_{\mathrm{c}}=138$ [16].

Finally, it should be noted that in Refs. [10, 11], they have used the same regularization for the Coulomb field of impurities, then an equation for the spectrum (up to the notation) in the case of $s=1$ had the form (57). However, results of numerical calculations refer to different physical parameters than in our consideration. Moreover, calculations of a critical charge were done only in the zero limit of the cutoff parameter. We note that a consideration of critical charges, similar to the present work, was undertaken in Refs. [8] and [9], where, however, the discrete spectrum was not investigated.

\section{Acknowledgements}

The work is supported by Russian Science Foundation (Grant No. 19-12-00042).

\section{References}

[1] G. W. Semenoff, Phys. Rev. Lett. 53, 2449 (1984)

[2] J. S. Goldstein, Phys. Rev. 91, 1516 (1953); P. I. Fomin, V. P. Gusynin, V . A. Miransky, et al., Rivista Nuovo Cimento6, 5 (1983); N. Setŏ, Prog. Theor. Phys. Suppl. 95, 25 (1988). 
[3] A. E. Shabad and V. V. Usov, Phys. Rev. Lett.96, 180401 (2006); Phys.Rev. D 73, 125021 (2006).

[4] S. P. Gavrilov, D. M. Gitman, and N. Yokomizo, Phys. Rev. D 86, 125022 (2012)

[5] N. Vandecasteele, A. Barreiro, M. Lazzeri, et al., Phys. Rev. B 82, 045416 (2010)

[6] K. Nomura and A. H. MacDonald, Phys. Rev. Lett. 98, 076602 (2007)

[7] V. N. Kotov, B. Uchoa, V. M. Pereira, et al., Rev. Mod. Phys. 84, 1067 (2012)

[8] V. M. Pereira, V. N. Kotov and A. C. Neto, Phys. Rev. B 78, 085101 (2008)

[9] W. Zhu, Z. Wang, Q. Shi, et al., Phys. Rev. B 79, 155430 (2009)

[10] E. V. Gorbar, V. P. Gusynin and O. O. Sobol, Low Temp. Phys. 44, 371-400 (2018)

[11] O. V. Gamayun, E. V. Gorbar and V. P. Gusynin, Phys. Rev. B 80, 165429 (2009)

[12] B. L. Voronov, D. M. Gitman and I. V. Tyutin, Theor. and Math. Phys. 150, 34-72 (2007)

[13] D. M. Gitman, I. V. Tyutin and B. L. Voronov, Self-adjoint Extensions in Quantum Mechanics: General Theory and Applications to Schrödinger and Dirac Equations with Singular Potentials, Birkäuser, New York, (2012)

[14] D. M. Gitman, A. D. Levin, I. V. Tyutin, et al., Phys. Scr. 87, 038104 (2013)

[15] V. M. Kuleshov, V. D. Mur, N. B. Narozhny, et al., Phys.-Usp. 58, 785 (2015)

[16] B. L. Voronov, D. M. Gitman, A. D. Levin, et al., Theor. and Math. Phys. 187, 633-648 (2016)

[17] J. González, F. Guinea and M. A. H. Vozmediano, Nucl. Phys. B 424, 595-618 (1994)

[18] T. Ando, J. of the Phys. Soc. of Japan 75, 074716 (2006)

[19] H. Raza (Ed.), Graphene Nanoelectronics. Metrology, Synthesis Properties, and Applications, Springer, New York, (2012)

[20] N. I. Akhiezer and I. M. Glazman, Theory of Linear Operators in in Hilbert space, Pitman, Boston, (1981)

[21] A. I. Akhiezer and V. B. Berestetskii, Elements of Quantum Electrodynamics, Israel Program for Sci. Tr., London, (1962)

[22] I. S. Gradshteyn and I. M. Ryzhik, Tables of Integrals, Series, and Products, Acad. Press, San Diego, (2014)

[23] A. Érdelyi, W. Magnus, F. Oberhettinger, et al., Higher Transcendental Functions Vol. 2, McGraw-Hill, New York, (1953) 
[24] M. I. Katsnelson, Graphene: carbon in two dimensions, Cambridge University Press, New York, (2012)

[25] Y. Wang, V. W. Brar, A. V. Shytov, et al., Nat. Phys. 8, 653 (2012)

[26] K. S. Gupta and S. Sen, Phys. Rev. B 78, 205429 (2008)

[27] V. R. Khalilov and K. E. Lee, Theor. and Math. Phys. 169 1683-1703 (2011)

[28] V. S. Popov, Sov. J. Nuc. Phys.-USSR 12, 235 (1971); Sov. Phys.-JETP 601228 (1971)

[29] Ya. B. Zel'dovich and V. S. Popov, Sov. Phys.-Usp. 14, 673 (1972) 